Dimitri P. Bertsekas · Paul Tseng

\title{
Set intersection theorems and existence of optimal solutions
}

Received: 14 December 2004 / Accepted: 25 January 2006 /

Published online: 13 May 2006

(C) Springer-Verlag 2006

\begin{abstract}
The question of nonemptiness of the intersection of a nested sequence of closed sets is fundamental in a number of important optimization topics, including the existence of optimal solutions, the validity of the minimax inequality in zero sum games, and the absence of a duality gap in constrained optimization. We consider asymptotic directions of a sequence of closed sets, and introduce associated notions of retractive, horizon, and critical directions, based on which we provide new conditions that guarantee the nonemptiness of the corresponding intersection. We show how these conditions can be used to obtain simple and unified proofs of some known results on existence of optimal solutions, and to derive some new results, including a new extension of the Frank-Wolfe Theorem for (nonconvex) quadratic programming.
\end{abstract}

Key words. Set intersection - Asymptotic direction - Recession direction - Global minimum - Frank-Wolfe theorem - Quasiconvex function

\section{Introduction}

In this paper, we focus on the question of whether a set intersection $\cap_{k=0}^{\infty} S_{k}$ is nonempty, where $\left\{S_{k}\right\}$ is a sequence of nonempty closed sets in $\mathbb{R}^{n}$ with $S_{k+1} \subset S_{k}$ for all $k$. This is a fundamental issue in optimization, because it lies at the heart of a number of important questions, such as the following:

1. Does a function $f: \mathbb{R}^{n} \mapsto(-\infty, \infty]$ attain a minimum over a set $X$ ? This is true if and only if the intersection

$$
\bigcap_{k=0}^{\infty}\left\{x \in X \mid f(x) \leq \gamma_{k}\right\}
$$

is nonempty, where $\left\{\gamma_{k}\right\}$ is a scalar sequence with $\gamma_{k} \downarrow \inf _{x \in X} f(x)$.

2. If $C$ is a closed set and $A$ is a matrix, is $A C$ closed? To prove this, we may let $\left\{y_{k}\right\}$ be a sequence in $A C$ that converges to some $\bar{y} \in \mathbb{R}^{n}$, and then show that $\bar{y} \in A C$. If we introduce the sets

$$
W_{k}=\left\{z \mid\|z-\bar{y}\| \leq\left\|y_{k}-\bar{y}\right\|\right\}, \quad N_{k}=\left\{x \mid A x \in W_{k}\right\},
$$

D. P. Bertsekas: Department of Electrical Engineering and Computer Science, M.I.T., Cambridge, MA 02139, USA

e-mail: dimitrib@mit.edu

P. Tseng: Department of Mathematics, University of Washington, Seattle, WA 98195, USA

e-mail: tseng@math.washington. edu

Mathematical subject classification (1991): 49J99 · 90C25 · 90C26 · 90C30 
and

$$
S_{k}=C \cap N_{k}
$$

it is sufficient to show that the intersection $\cap_{k=0}^{\infty} S_{k}$ is nonempty.

3. Given a function $F: \mathbb{R}^{n+m} \mapsto(-\infty, \infty]$ that is closed (i.e. has a closed epigraph), is the function $f: \mathbb{R}^{n} \mapsto[-\infty, \infty]$ defined by $f(x)=\inf _{z \in \mathbb{R}^{m}} F(x, z)$ closed? It is known that this is a critical question in duality theory and minimax theory (see, e.g. $[1,11,25])$. Properties of epi $(f)$, the epigraph of $f$, can be inferred from properties of epi $(F)$, the epigraph of $F$, by using the relation

$$
P(\operatorname{epi}(F)) \subset \operatorname{epi}(f) \subset \operatorname{cl}(P(\operatorname{epi}(F))),
$$

where $\operatorname{cl}(\cdot)$ denotes closure of a set and $P(\cdot)$ denotes projection on the space of $(x, w)$, i.e. $P(x, z, w)=(x, w)$. [The left-hand side of this relation follows from the definition

$$
\operatorname{epi}(f)=\left\{(x, w) \mid \inf _{z \in \mathbb{R}^{m}} F(x, z) \leq w\right\} .
$$

To show the right-hand side, note that for any $(x, w) \in \operatorname{epi}(f)$ and every $k$, there exists a $z_{k}$ such that $\left(x, z_{k}, w+1 / k\right) \in \operatorname{epi}(F)$, so that $(x, w+1 / k) \in P(\operatorname{epi}(F))$ and $(x, w) \in \operatorname{cl}(P(\operatorname{epi}(F)))]$. If $F$ is closed and we can show that the projection $P(\cdot)$ preserves closedness [a special case of question (2) above], it follows that epi $(f)$ is closed and $f$ is closed.

If the sets $S_{k}$ are compact, then $\cap_{k=0}^{\infty} S_{k}$ is nonempty and compact, a fact that underlies Weierstrass' theorem (a closed function $f$ attains a minimum over a compact set $X$ ); see the reasoning in (1) above. The special case where the sets $S_{k}$ are convex has been the subject of much research, following the work of Helly [18] and others (e.g. Fenchel [17] and Rockafellar [25]). A recent line of analysis that focuses on the issues (1)-(3) discussed above, is given in Sect. 1.5 of Bertsekas et al. [11], and is based on the notions of a direction of recession and lineality space. In this paper, we develop conditions that guarantee the nonemptiness of the intersection $\cap_{k=0}^{\infty} S_{k}$ in the general case where the sets $S_{k}$ may not be bounded and may not be convex.

Our analysis is based on an extension of the notion of a direction of recession, the notion of an asymptotic direction of the set sequence $\left\{S_{k}\right\}$, which is a nonzero vector in the horizon limit of $\left\{S_{k}\right\}$ studied by Rockafellar and Wets [27]. A related notion of a retractive direction (see Sect. 2) is new in the form given here, but is closely related to ideas developed, principally within the context of optimization, by Auslender; see, e.g. [2-4], and the book by Auslender and Teboulle [1]. These sources focus on asymptotic directions of sets and functions, rather than sequences of sets. It appears that the notion of asymptotic direction of a sequence of sets (rather than a set or a function) is simpler and often more convenient for the aforementioned optimization applications. We also develop the notions of a horizon and critical directions, which are formulated here for the first time, for both cases of a single set and a sequence of sets. These notions are used to study some important cases where the sets $S_{k}$ have asymptotic directions that are not retractive; e.g. when $S_{k}$ are specified by quadratic inequalities. We show that asymptotic, retractive, horizon, and critical directions provide the basis for new set intersection theorems, new existence of optimal solutions results, and simpler and unified proofs of 
known results. Some related analysis is given in Tseng and Ozdaglar [29], where variants of asymptotic direction and retractive direction are used to derive generalizations of certain existence results by Auslender and by Luo and Zhang [21].

We note that in the case where the sets $S_{k}$ are convex, as well as closed, the set of asymptotic directions of $\left\{S_{k}\right\}$ is in effect the intersection of the recession cones of the sets $S_{k}$, and the set of retractive directions is related (but is not equal) to the intersection of the lineality spaces of the sets $S_{k}$. A horizon direction of $\left\{S_{k}\right\}$ is also somewhat related to common directions of recession of the sets $S_{k}$ (see the discussion of Sect. 3).

We note also that the set of asymptotic directions, when specialized to a closed, possibly nonconvex, set (rather than a nested sequence of closed sets), is essentially the horizon cone described by Rockafellar and Wets [27], and the asymptotic cone described by Auslender and Teboulle [1]. These cones have been introduced in the works of Dedieu $[13,14]$, and have been the subject of considerable attention recently; see the references in $[1,27]$.

We organize the material as follows. In Sect. 2, we introduce asymptotic directions and retractive directions, and we prove a key result relating to the nonemptiness of a closed set intersection. In Sect. 3, we introduce horizon and critical directions, and we use them to derive nonemptiness results for the case where $S_{k}$ is the intersection of a finite number of closed sets. Some of these results relate to level sets of bidirectionally flat functions, a class that includes convex quadratic and, more generally, convex polynomial functions. Finally, in Sect. 4 we unify and extend various known results on the existence of optimal solutions, including a generalization of the Frank-Wolfe Theorem of (nonconvex) quadratic programming. While we do not discuss in detail the application to questions of preservation of closedness under linear transformation and partial minimization, our results can also be used for the analysis of these issues, as discussed earlier (see also some discussion at the end of Sect. 2).

Throughout the paper, all analysis is done in the $n$-dimensional Euclidean $\mathbb{R}^{n}$. Thus, unless otherwise specified, vectors and subsets are from $\mathbb{R}^{n}$. All vectors are viewed as columns vectors, and a prime denotes transpose. The standard Euclidean norm, $\|x\|=$ $\sqrt{x^{\prime} x}$ is used throughout.

\section{Asymptotic directions and retractive directions}

We first introduce the notion of asymptotic direction of a nested set sequence $\left\{S_{k}\right\}$, i.e. a sequence such that $S_{k+1} \subset S_{k}$ for all $k$.

Definition 1. Let $\left\{S_{k}\right\}$ be a nested sequence of nonempty closed sets. We say that a nonzero vector $d$ is an asymptotic direction of $\left\{S_{k}\right\}$ if there exists a sequence $\left\{x_{k}\right\}$ such that

$$
x_{k} \in S_{k}, \quad k=0,1, \ldots, \quad\left\|x_{k}\right\| \rightarrow \infty, \quad \frac{x_{k}}{\left\|x_{k}\right\|} \rightarrow \frac{d}{\|d\|} .
$$

A sequence $\left\{x_{k}\right\}$ associated with an asymptotic direction $d$ as above is called an asymptotic sequence corresponding to $d$. An asymptotic direction d of $\left\{S_{k}\right\}$ is called retractive if, for every corresponding asymptotic sequence $\left\{x_{k}\right\}$, there exists an integer $\bar{k}$ such that

$$
x_{k}-d \in S_{k}, \quad \forall k \geq \bar{k}
$$

The set sequence $\left\{S_{k}\right\}$ is called retractive if all its asymptotic directions are retractive. 
Roughly speaking, an asymptotic direction is a direction along which we can escape towards $\infty$ through each of the sets $S_{k}$ (see Fig. 1). In particular, $\left\{S_{k}\right\}$ has an asymptotic direction if and only if all the sets $S_{k}$ are unbounded. Equivalently, a nonzero vector $d$ is an asymptotic direction of $\left\{S_{k}\right\}$ if there exists a sequence $\left\{x_{k}\right\}$ and a positive sequence $\left\{t_{k}\right\}$ with $x_{k} \in S_{k}$ for all $k, t_{k} \rightarrow \infty$, and $x_{k} / t_{k} \rightarrow d$. It can be seen that the asymptotic directions are exactly the nonzero vectors in the horizon limit of $\left\{S_{k}\right\}$, as defined in [27] (see Sect. 4F). A retractive asymptotic direction $d$ is one whose asymptotic sequences still belong to the corresponding sets $S_{k}$ when shifted by $-d$ (see Fig. 2). The importance of retractive directions is illustrated by the following key proposition, showing that the intersection of a retractive nested sequence of nonempty closed sets $S_{k}, k=0,1, \ldots$, is nonempty. Its proof uses the argument that if this intersection is empty, then the sequence of minimum norm vectors $x_{k}$ of $S_{k}$ would be unbounded and each cluster point $d$ of $x_{k} /\left\|x_{k}\right\|$ would be an asymptotic direction, so moving $x_{k}$ opposite of $d_{k}$ would yield another point in $S_{k}$ of lesser norm than $x_{k}$, contradicting $x_{k}$ having minimum norm.

Proposition 1. A retractive nested sequence of nonempty closed sets has nonempty intersection.

Proof. Let $\left\{S_{k}\right\}$ be the given sequence. For each $k$, let $x_{k}$ be a vector of minimum norm on the closed set $S_{k}$ (such a vector exists by Weierstrass' theorem, since it can be obtained by minimizing $\|x\|$ over all $x$ in the compact set $S_{k} \cap\left\{x \mid\|x\| \leq\left\|\bar{x}_{k}\right\|\right\}$, where $\bar{x}_{k}$ is any vector in $S_{k}$ ). It will be sufficient to show that a subsequence $\left\{x_{k}\right\}_{k \in \mathcal{K}}$ is bounded. Then, since $\left\{S_{k}\right\}$ is nested, for each $m$, we have $x_{k} \in S_{m}$ for all $k \in \mathcal{K}, k \geq m$, and since $S_{m}$ is closed, each of the limit points of $\left\{x_{k}\right\}_{k \in \mathcal{K}}$ will belong to each $S_{m}$ and hence also to $\cap_{m=0}^{\infty} S_{m}$, thereby showing the result. Thus, we will prove the proposition by showing that there is no subsequence of $\left\{x_{k}\right\}$ that is unbounded.

Indeed, assume the contrary, let $\left\{x_{k}\right\}_{k \in \mathcal{K}}$ be a subsequence such that

$$
\lim _{k \rightarrow \infty, k \in \mathcal{K}}\left\|x_{k}\right\|=\infty
$$

and let $d$ be the limit of a subsequence $\left\{x_{k} /\left\|x_{k}\right\|\right\}_{k \in \overline{\mathcal{K}}}$, where $\overline{\mathcal{K}} \subset \mathcal{K}$. For each $k=$ $0,1, \ldots$, define $y_{k}=x_{m}$, where $m$ is the smallest index $m \in \overline{\mathcal{K}}$ with $k \leq m$. Then, since $y_{k} \in S_{k}$ for all $k$ and $\lim _{k \rightarrow \infty}\left\{y_{k} /\left\|y_{k}\right\|\right\}=d$, we see that $d$ is an asymptotic direction of $\left\{S_{k}\right\}$ and $\left\{y_{k}\right\}$ is an asymptotic sequence corresponding to $d$. Using the retractiveness assumption, let $\bar{k}$ be such that $y_{k}-d \in S_{k}$ for all $k \geq \bar{k}$. We have $d^{\prime} y_{k} \rightarrow \infty$ since $d^{\prime} y_{k} /\left\|y_{k}\right\| \rightarrow\|d\|^{2}=1$, so for all $k \geq \bar{k}$ with $2 d^{\prime} y_{k}>1$, we obtain

$$
\left\|y_{k}-d\right\|^{2}=\left\|y_{k}\right\|^{2}-\left(2 d^{\prime} y_{k}-1\right)<\left\|y_{k}\right\|^{2} \text {. }
$$

This is a contradiction, since for infinitely many $k, y_{k}$ is the vector of minimum norm on $S_{k}$.

For an example where the above proposition applies, consider the sequence $\left\{S_{k}\right\}$ of Fig. 2a. Here the asymptotic directions $(0, \beta), \beta>0$, are retractive, and indeed the intersection $\cap_{k=0}^{\infty} S_{k}$ is nonempty. On the other hand, the condition of the proposition is far from necessary for nonemptiness of $\cap_{k=0}^{\infty} S_{k}$. For example, the sequence $\left\{S_{k}\right\}$ of 


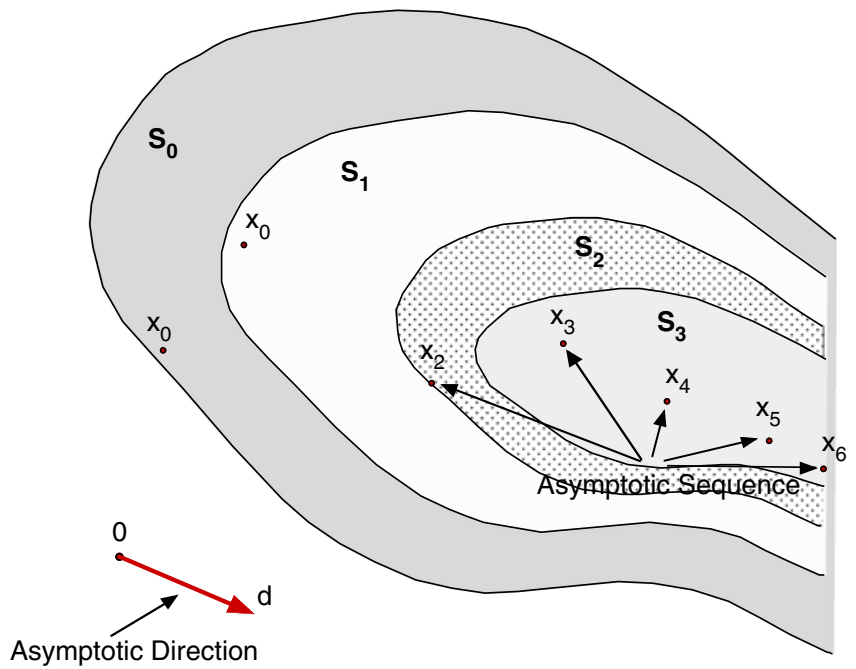

Fig. 1 Illustration of an asymptotic direction of a sequence of nonconvex sets and a corresponding asymptotic sequence. The normalized direction sequence $x_{k} /\left\|x_{k}\right\|$ must converge to the unit direction $d /\|d\|$

Fig. $2 \mathrm{~b}$ has nonempty intersection, yet the asymptotic directions $(0, \beta), \beta>0$, are not retractive.

We note that the conclusion of the preceding proposition also holds under a weaker definition of retractive direction, whereby $d$ is called retractive if, for every corresponding asymptotic sequence $\left\{x_{k}\right\}$, there exist a bounded sequence of positive scalars $\left\{\alpha_{k}\right\}$ and an index $\bar{k}$ such that $x_{k}-\alpha_{k} d \in S_{k}$ for all $k \geq \bar{k}$. This definition does not work well, however, when we consider intersections of two or more sequences. By contrast, under the given Definition 1, it follows that the sequence obtained by intersection or union or Cartesian product of two retractive sequences is retractive. In particular, we have the following proposition.

Proposition 2. Let $\left\{S_{k}^{j}\right\}, j=1, \ldots, r$, be retractive nested sequences of nonempty closed sets. Then the sequences $\left\{U_{k}\right\}$ and $\left\{T_{k}\right\}$, where

$$
\begin{array}{ll}
U_{k}=S_{k}^{1} \cup S_{k}^{2} \cup \cdots \cup S_{k}^{r}, & k=0,1, \ldots, \\
T_{k}=S_{k}^{1} \times S_{k}^{2} \times \cdots \times S_{k}^{r}, & k=0,1, \ldots,
\end{array}
$$

are both retractive. If the sets

$$
N_{k}=S_{k}^{1} \cap S_{k}^{2} \cap \cdots \cap S_{k}^{r}, \quad k=0,1, \ldots,
$$

are all nonempty, then $\left\{N_{k}\right\}$ is retractive.

\subsection{Asymptotic directions of closed sets}

We now specialize the definitions of asymptotic directions and retractiveness to the case where all the sets in the sequence are the same. For this case, the notion of asymptotic direction was studied in the works of Dedieu [13, 14], and others [1, 27]. 


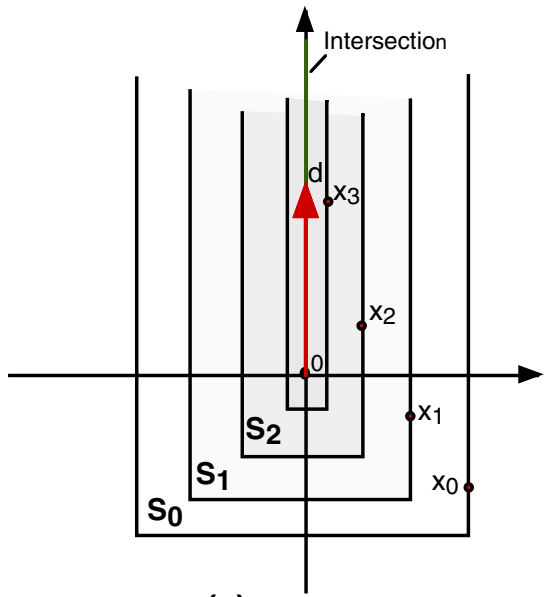

(a) Retractive

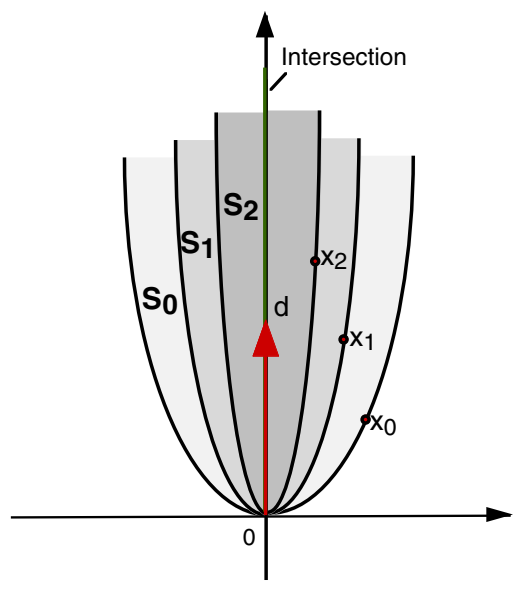

(b) Nonretractive

Fig. 2 Illustration of retractive and nonretractive directions in $\mathbb{R}^{2}$. In both cases, the set of asymptotic directions is $\{(0, \beta) \mid \beta>0\}$, and the intersection of the corresponding sequence is the set $\left\{\left(0, x_{2}\right) \mid x_{2} \geq 0\right\}$. In (a), we have

$$
S_{k}=\left\{\left(x_{1}, x_{2}\right)|\quad| x_{1} \mid \leq \frac{1}{k+1}, \quad x_{2} \geq-\frac{1}{k+1}\right\},
$$

and it can be seen that every asymptotic direction is retractive. In (b), we have

$$
S_{k}=\left\{\left(x_{1}, x_{2}\right) \mid x_{2} \geq(k+1) x_{1}^{2}\right\},
$$

and it can be seen that all asymptotic directions are not retractive. As an example, for the asymptotic direction $(0,1)$, the corresponding asymptotic sequence $\left\{\left(k,(k+1) k^{2}\right)\right\}$ does not belong to $S_{k}$ when shifted by $(0,-1)$

Definition 2. Given a nonempty closed set $S$, we say that a nonzero vector $d$ is an asymptotic direction of $S$ if it is an asymptotic direction of the sequence $\left\{S_{k}\right\}$, where $S_{k}=S$ for all $k$, i.e. there exists a sequence $\left\{x_{k}\right\} \subset S$, called an asymptotic sequence corresponding to $d$, such that

$$
\left\|x_{k}\right\| \rightarrow \infty \text { and } \frac{x_{k}}{\left\|x_{k}\right\|} \rightarrow \frac{d}{\|d\|} .
$$

An asymptotic direction $d$ is called retractive if, for every corresponding asymptotic sequence $\left\{x_{k}\right\}$, there exists an integer $\bar{k}$ such that

$$
x_{k}-d \in S, \quad \forall k \geq \bar{k}
$$

The set $S$ is called retractive if all its asymptotic directions $d$ are retractive.

We note that our notion of a retractive set is equivalent to the notion of an asymptotically linear set introduced by Auslender [4], and Auslender and Teboulle [1] (see [1], p. 37), who provide a great deal of related analysis, including the corresponding version of Proposition 2. 
For any nonempty closed set $S$, let $A_{S}$ denote the set of asymptotic directions of $S$. The next proposition shows that the asymptotic directions of a closed set sequence are the common asymptotic directions of the sets in the sequence. This proposition can be deduced from Exercise 4.21 in [27] about the horizon limit of a set sequence. We include a proof for completeness.

Proposition 3. Let $\left\{S_{k}\right\}$ be a nested sequence of nonempty closed sets. Then $d$ is an asymptotic direction of $\left\{S_{k}\right\}$ if and only if $d \in \cap_{k=0}^{\infty} A_{S_{k}}$.

Proof. Let $d$ belong to $\cap_{k=0}^{\infty} A_{S_{k}}$. For each $k$, since $d \in A_{S_{k}}$, there exists an $x_{k} \in S_{k}$ such that $\left\|x_{k}\right\| \geq k$ and $\left|x_{k} /\left\|x_{k}\right\|-d /\|d\|\right| \leq 1 / k$. Then $\left\|x_{k}\right\| \rightarrow \infty$ and $x_{k} /\left\|x_{k}\right\| \rightarrow d /\|d\|$, showing that $d$ is an asymptotic direction of $\left\{S_{k}\right\}$.

Conversely, let $d$ be an asymptotic direction of $\left\{S_{k}\right\}$, and let $\left\{x_{k}\right\}$ be a corresponding asymptotic sequence. Then, for each $\bar{k}$, we have $x_{k} \in S_{k} \subset S_{\bar{k}}$ for all $k \geq \bar{k}$. This shows that $d \in A_{S_{\bar{k}}}$. Since this is true for each $\bar{k}$, we have $d \in \cap_{k=0}^{\infty} A_{S_{k}}$.

For any nonempty set $S$, we say that a vector $d$ is a (global) direction of recession of $S$ if $x+\alpha d \in S$ for all $x \in S$ and $\alpha \geq 0$ (see, e.g. [11, 25, 27]). The set of all directions of recession of $S$, denoted by $R_{S}$, is the recession cone of $S$. It is well known that, for a nonempty closed convex set, the set of asymptotic directions coincides with the set of nonzero directions of recession (see [27], Theorem 3.6 and Sect. 6G). The lineality space of $S$ is defined as

$$
L_{S}=R_{S} \cap\left(-R_{S}\right) .
$$

It is readily seen that the lineality space directions are retractive.

The following result, which does not seem to have been reported in the literature, shows that the complement of an open convex set is retractive.

Proposition 4. Let $S$ be a nonempty closed set that is the complement of an open convex set. Then, $S$ is retractive.

Proof. Assume, to arrive at a contradiction, that there exists an asymptotic direction $d$ of $S$ that is not retractive, and let $\left\{x_{k}\right\}$ be a corresponding asymptotic sequence for which the definition of retractiveness is violated. Then $x_{k}-d \notin S$ for an infinite subsequence of indexes $k$. Without loss of generality, assume that this is true for all indexes $k$. We have $x_{k} /\left\|x_{k}\right\| \rightarrow d /\|d\|$ and $\left\|x_{k}\right\| \rightarrow \infty$, so that $\left\|x_{k}-d\right\| \rightarrow \infty$ and $\left(x_{k}-d\right) /\left\|\left(x_{k}-d\right)\right\| \rightarrow d /\|d\|$. It follows that $\left\{x_{k}-d\right\}$ is an asymptotic sequence of the closure of the convex set $\mathbb{R}^{n} \backslash S$, and $d$ is the corresponding asymptotic direction. Then $d$ is a direction of recession of this closed convex set (see [27], Theorem 3.6). Since $x_{k}-d$ lies in $\mathbb{R}^{n} \backslash S$, the interior of this set, all the vectors of the form $x_{k}+\alpha d$ with $\alpha \in[-1, \infty)$, including $x_{k}$, lie in $\mathbb{R}^{n} \backslash S$, contradicting the hypothesis $x_{k} \in S$.

The preceding proposition can be extended to more general convex sets by restricting attention to their affine hull. In particular, if $C$ is a relatively open convex set (i.e. a convex set that coincides with its relative interior), then the intersection of the complement of $C$ with the affine hull of $C$ is retractive. Note that by Proposition 4, a closed half-space is retractive since its complement is an open half-space, which is convex. 
It follows from Proposition 2 that a polyhedral set is retractive, since it is the intersection of a finite number of half-spaces. More generally, the intersection and union of a finite number of complements of open convex sets is retractive. An example of such a set is

$$
S=\{x \mid f(x) \geq 0\},
$$

where $f$ is the pointwise minimum of a finite number of real-valued convex functions. Note that the finiteness assumption is necessary for retractiveness, and in particular a nonpolyhedral closed convex cone need not be retractive (see the example given at the end of Sect. 2).

It is well known that a polyhedral set $S$ can be expressed as the vector sum of a compact polyhedral set and a polyhedral cone $N$ (see, e.g. [11,25]). Moreover, the asymptotic directions of $S$ are exactly the nonzero vectors in $N$. The following proposition presents a useful generalization of these facts.

Proposition 5. Let $S$ be a set which is the vector sum of a compact set and a closed set $N$. Then the asymptotic directions of $S$ are exactly the asymptotic directions of $N$, and $S$ is retractive if and only if $N$ is retractive.

Proof. We write $S=\bar{S}+N$, where $\bar{S}$ is nonempty and compact. Let $d$ be an asymptotic direction of $N$. Then there exists a sequence $\left\{y_{k}\right\} \subset N$ with $\left\|y_{k}\right\| \rightarrow \infty$ and $y_{k} /\left\|y_{k}\right\| \rightarrow d /\|d\|$. Fix any $\bar{x} \in \bar{S}$ and let

$$
x_{k}=\bar{x}+y_{k}, \quad k=0,1, \ldots,
$$

Then $x_{k} \in S,\left\|x_{k}\right\| \rightarrow \infty$, and $x_{k} /\left\|x_{k}\right\| \rightarrow d /\|d\|$, showing that $d$ is an asymptotic direction of $S$.

Conversely, let $d$ be an asymptotic direction of $S$. Then, there exists a sequence $\left\{x_{k}\right\} \subset S$ with $\left\|x_{k}\right\| \rightarrow \infty$ and $x_{k} /\left\|x_{k}\right\| \rightarrow d /\|d\|$. We can represent $x_{k}$ as

$$
x_{k}=\bar{x}_{k}+y_{k}, \quad \forall k=0,1, \ldots,
$$

where $\bar{x}_{k} \in \bar{S}$ and $y_{k} \in N$. Since $\bar{S}$ is compact, $\left\{\bar{x}_{k}\right\}$ is bounded. This implies $\left\|y_{k}\right\| \rightarrow \infty$ and $y_{k} /\left\|y_{k}\right\| \rightarrow d /\|d\|$, showing that $d$ is an asymptotic direction of $N$.

We note that $d$ is an asymptotic direction of $S$, with $\left\{x_{k}\right\}$ being a corresponding asymptotic sequence, if and only if $x_{k}=\bar{x}_{k}+y_{k}$, where $\left\{\bar{x}_{k}\right\} \subset \bar{S}$ and $\left\{y_{k}\right\}$ is an asymptotic sequence of $N$ corresponding to $d$, viewed as an asymptotic direction of $N$. Hence $d$ is retractive, when viewed as an asymptotic direction of $S$ if and only if $d$ is retractive when viewed as an asymptotic direction of $N$.

By Proposition 2 and 5, the asymptotic directions of a nonempty set of the form $S=S^{1} \cap \cdots \cap S^{m}$ such that each of the sets $S^{i}, i=1, \ldots, m$, is the vector sum of a compact set and a polyhedral cone $N^{i}$, are the nonzero vectors in $\cap_{i=1}^{m} N^{i}$. Furthermore, $S$ is retractive. Similarly, the vector sum of a compact set and the union of a finite number of polyhedral cones is retractive. Sets of this type have been studied within the class of asymptotically polyhedral sets introduced by Auslender and Teboulle [1], who prove a similar result, namely that they are asymptotically linear (see [1], Proposition 2.3.1).

By using Proposition 1 and 3, we have the following new set intersection results involving asymptotic directions and lineality space. 
Proposition 6. Let $\left\{C_{k}\right\}$ be a nested sequence of nonempty closed sets. Denote

$$
A=\cap_{k=0}^{\infty} A_{C_{k}}, \quad L=\cap_{k=0}^{\infty} L_{C_{k}} .
$$

(a) If $A \subset L$, then $\left\{C_{k}\right\}$ is retractive, and $\cap_{k=0}^{\infty} C_{k}$ is nonempty.

(b) Let $X$ be a retractive closed set. Assume that all the sets $S_{k}=X \cap C_{k}$ are nonempty, and that

$$
A_{X} \cap A \subset L .
$$

Then, $\left\{S_{k}\right\}$ is retractive, and $\cap_{k=0}^{\infty} S_{k}$ is nonempty.

Proof. (a) This is a special case of (b) whereby we take $X=\mathbb{R}^{n}$, so that $A_{X}$ is the set of nonzero vectors in $\mathbb{R}^{n}$ and $A_{X} \cap A=A$.

(b) By Proposition 3, an asymptotic direction $d$ of $\left\{S_{k}\right\}$ must belong to $A_{X} \cap A$, and hence also to $L$. Thus, for any corresponding asymptotic sequence $\left\{x_{k}\right\}$, we have $x_{k} \in C_{k}$ and hence $x_{k}-d \in C_{k}$ for all $k$. Since $d$ is an asymptotic direction of $X$ and $X$ is retractive, this implies that $d$ is retractive for $\left\{S_{k}\right\}$. By Proposition $1, \cap_{k=0}^{\infty} S_{k}$ is nonempty.

If all the sets $C_{k}$ are convex, then $A_{C_{k}}$ comprises the nonzero vectors in $R_{C_{k}}$, and since $R_{C_{k}} \supset L_{C_{k}}$, we have $A \supset L \backslash\{0\}$, and the assumption $A \subset L$ in part (a) of Proposition 6 is equivalent to $A=L \backslash\{0\}$. In this case, part (a) and the special case where $X$ is a polyhedral set in part (b) are known (see [11] and the references there in), but the proof given here is simpler than those found in the literature. Part (b) applies more generally to the case where $X$ is the vector sum of a compact set and a set $N$, with $N$ being the intersection and union of a finite number of polyhedral cones and complements of open convex sets (see Propositions 2, 4, and 5).

Regarding the preservation of closedness of a set $C$ under a linear transformation $A$, by applying Propositions 1 and 2, we obtain that $A C$ is closed if $C$ is a closed retractive set [we use here the retractiveness of the sequence $\left\{N_{k}\right\}$ of Eq. (1)]. This result has been shown by Auslender and Teboulle, but using a more specialized argument (see [1], Theorems 2.3.1 and 2.3.3). A very different condition for closedness of $A C$ was given by Pataki [23], who assumes $C$ to be a "nice" closed convex cone (in particular, a polyhedral cone, the second-order cone, or the cone of symmetric positive semidefinite matrices) in addition to a technical constraint qualification involving the range of $A^{\prime}$, the dual cone $C^{*}$, and the closure of the set of feasible directions of $C^{*}$. Pataki's result is different in nature from ours, since it can be seen that neither the second-order cone, which is the epigraph of the Euclidean norm function, nor the cone of $n \times n$ symmetric positive semidefinite matrices are retractive. For example, $d=(1,0,1)$ is an asymptotic direction of the second-order cone $C=\{(u, v, w) \mid\|(u, v)\| \leq w\}$, with corresponding asymptotic sequence $x_{k}=\left(k, \sqrt{k}, \sqrt{k^{2}+k}\right)$, but $x_{k}-d \notin C$ for all $k=1,2, \ldots$

\section{Horizon directions and associated intersection theorems}

While retractiveness is clearly an important property, it is inadequate for proving or explaining some of the principal set intersection results that are useful in optimization. 
Prominent among these, are results relating to sets specified by quadratic inequalities. In this section, we introduce some additional properties of asymptotic directions, which together with retractiveness, can be used to provide a more complete framework for set intersection analysis.

We will focus on a key question, which does not seem to have been addressed so far in the literature: Given two nested set sequences $\left\{S_{k}^{1}\right\}$ and $\left\{S_{k}^{2}\right\}$ each with nonempty intersection by itself, and with

$$
S_{k}^{1} \cap S_{k}^{2} \neq \emptyset, \quad k=0,1, \ldots,
$$

what causes the intersection sequence $\left\{S_{k}^{1} \cap S_{k}^{2}\right\}$ to have an empty intersection? By sketching a few examples (see Fig. 3), one may suspect that the trouble lies with the existence of some "critical asymptotes." Roughly, these are asymptotic directions $d$, common to $\left\{S_{k}^{1}\right\}$ and $\left\{S_{k}^{2}\right\}$, and such that starting at $\cap_{k} S_{k}^{2}$ (or $\cap_{k} S_{k}^{1}$ ) and moving along $d$, we do not meet $\cap_{k} S_{k}^{1}$ (or $\cap_{k} S_{k}^{2}$, respectively). With this in mind, we introduce a subset of asymptotic directions, called horizon directions, which we will subsequently use to make precise the meaning of a "critical asymptote".

Definition 3. Given a nested closed set sequence $\left\{S_{k}\right\}$ with nonempty intersection, we say that an asymptotic direction $d$ of $\left\{S_{k}\right\}$ is a horizon direction with respect to a set $G$ if, for every $x \in G$, there exists a scalar $\bar{\alpha} \geq 0$ such that $x+\alpha d \in \cap_{k=0}^{\infty} S_{k}$ for all $\alpha \geq \bar{\alpha}$. We say that $d$ is a global horizon direction if $G=\mathbb{R}^{n}$, and we say that it is a local horizon direction if $G=\cap_{k=0}^{\infty} S_{k}$.

Thus $d$ is a horizon direction with respect to $G$ if starting at any point of $G$ and going along $d$ we eventually enter and stay in $\cap_{k=0}^{\infty} S_{k}$. The definition of a horizon direction of a set sequence specializes naturally to the case of single closed set $S$ by viewing the set as the constant sequence of sets $\left\{S_{k}\right\}$, where $S_{k}=S$ for all $k$. Thus, e.g. the statement that $d$ is a horizon direction of $S$ with respect to $G$ means that $d$ is a horizon direction of the sequence $\left\{S_{k}\right\}$ with respect to $G$, where $S_{k}=S$ for all $k$.

It can be seen that if the sets $S_{k}$ are convex, the set of local horizon directions is equal to the set of asymptotic directions, and also to the set of nonzero common directions of recession of all the sets $S_{k}$ (see Proposition 3). The set of global horizon directions may be a strict subset of the set of asymptotic directions, even if the sets $S_{k}$ are convex (take, e.g. all the sets $S_{k}$ to be equal to the same line on the plane).

Note also that if $\left\{S_{k}^{1}\right\}$ and $\left\{S_{k}^{2}\right\}$ are nested closed set sequences such that the sequence $\left\{S_{k}^{1} \cap S_{k}^{2}\right\}$ has nonempty intersection, then a vector which is a horizon direction of both $\left\{S_{k}^{1}\right\}$ and $\left\{S_{k}^{2}\right\}$ with respect to a (common) set $G$ is also a horizon direction of $\left\{S_{k}^{1} \cap S_{k}^{2}\right\}$ with respect to $G$. However, the converse is not true, as simple examples indicate. On the other hand, the set of vectors that are global horizon directions of both $\left\{S_{k}^{1}\right\}$ and $\left\{S_{k}^{2}\right\}$ coincides with the set of global horizon directions of $\left\{S_{k}^{1} \cap S_{k}^{2}\right\}$.

Here are some examples illustrating horizon directions.

Example 1. Let $S$ be the complement of a bounded open set. Then all nonzero directions are asymptotic directions as well as global horizon directions. 


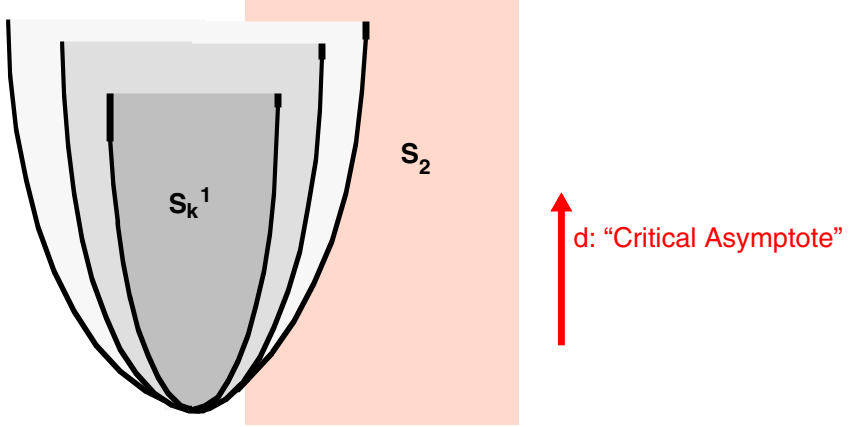

Fig. 3 Illustration of a set sequence $\left\{S_{k}^{1}\right\}$ and a set $S^{2}$ with

$$
S_{k}^{1} \cap S^{2} \neq \emptyset, \quad k=0,1, \ldots,
$$

and $\cap_{k=0}^{\infty} S_{k}^{1} \cap S^{2}=\emptyset$. The problem is that there exists some "critical asymptote" along which $\left\{S_{k}^{1}\right\}$ and $S^{2}$ "asymptotically lose contact"

Example 2. Let $f: \mathbb{R}^{n} \mapsto \mathbb{R}$ be a convex function that is coercive, and let $S=$ $\{x \mid f(x) \geq \gamma\}$ where $\gamma$ is a scalar. Then $S$ is closed and nonempty (since $f$ is realvalued and hence continuous over $\mathbb{R}^{n}$, as well as coercive), and because the complement of $S$ is bounded (by the coercivity of $f$ ), all nonzero directions are asymptotic directions as well as global horizon directions, as per the preceding example.

Example 3. (Vector sums of compact sets and polyhedral cones) Let $S=X_{1} \cap X_{2} \cap$ $\cdots \cap X_{m}$, where each $X_{i}$ is the vector sum of a compact set and a polyhedral cone $N_{i}$. Then, the set of asymptotic directions, which are the nonzero vectors in $\cap_{i=1}^{m} N_{i}$, is also equal to the set of local horizon directions. However, the set of global horizon directions may be strictly smaller, and in fact may be empty, even if $\cap_{i=1}^{m} N_{i}$ contains a nonzero direction (take, e.g. $m=1$ and $X_{1}$ to be a half-line on the plane).

Let us introduce a class of convex functions that includes convex quadratic and, more generally, convex polynomial functions. These functions were introduced in Exercise 2.7 of [11], and were shown to be interesting within the contexts of set intersection, existence of optimal solutions, and strong duality (see [11], Proposition 6.5.7). We recall that, for a closed proper convex function $f: \mathbb{R}^{n} \mapsto(-\infty, \infty]$, the recession cone of $f$ and the constancy space of $f$, denoted $R_{f}$ and $L_{f}$, respectively, are the (common) recession cone and lineality space of its nonempty level sets (see, e.g. [11, 25]). Moreover, a direction $d$ belongs to the recession cone $R_{f}$ if and only if, for every $x \in \operatorname{dom}(f)$, we have

$$
\lim _{\alpha \rightarrow \infty} \frac{f(x+\alpha d)}{\alpha} \leq 0
$$

(see [25], Theorems 8.5, 8.6).

Example 4. (Bidirectionally Flat Convex Functions) Let $f: \mathbb{R}^{n} \mapsto(-\infty, \infty]$ be a closed proper convex function with the property that a direction $d$ belongs to the 
constancy space $L_{f}$ if and only if

$$
\lim _{\alpha \rightarrow \infty} \frac{f(x+\alpha d)}{\alpha}=0, \quad \forall x \in \operatorname{dom}(f) .
$$

Functions of this type will be referred to as bidirectionally flat. It is clear that convex polynomial functions, including convex quadratic functions, are bidirectionally flat, since they are polynomial along any direction, and hence constant along any direction $d$ satisfying Eq. (2). Another class of bidirectionally flat functions, which also includes convex quadratic functions, have the form

$$
f(x)=h(A x)+c^{\prime} x+b,
$$

where $A$ is an $m \times n$ matrix, $c$ is a vector, $b$ is a scalar, and $h: \mathbb{R}^{m} \mapsto(-\infty, \infty]$ is a closed proper convex function satisfying

$$
\liminf _{\|y\| \rightarrow \infty} \frac{h(y)}{\|y\|}=\infty .
$$

In view of this property, we see that Eq. (2) is satisfied if and only if $d$ is in the nullspace of $A$ and $c^{\prime} d=0$, which is true if and only if $d \in L_{f}$.

A bidirectionally flat function is different from a weakly analytic convex function [20] (see also [5]), [1], which has the property of being constant along a line whenever it is constant along any open segment of the line. It is also different from a faithfully convex function [26], which has the property of being affine along a line whenever it is affine along any open segment of the line. In particular, the exponential function $f(x)=e^{x}$ is weakly analytic and faithfully convex, but not bidirectionally flat; the function $f(x)=\max \{0, x-1,-x-1\}$ is bidirectionally flat, but neither weakly analytic nor faithfully convex. We note that weakly analytic and faithfully convex functions also include convex polynomial functions as special cases, and play important roles in ensuring zero duality gap (rather than solution existence) in convex programs.

Let $\left\{S_{k}\right\}$ be a set sequence defined by the level sets of $f$ :

$$
S_{k}=\left\{x \mid f(x) \leq \gamma_{k}\right\},
$$

where $\left\{\gamma_{k}\right\}$ is a scalar nonnegative sequence with $\gamma_{k} \downarrow 0$, and such that all the sets $S_{k}$ are nonempty. Then, for any nonzero vector $d \in R_{f}$, one of the following two cases holds:

(1) $d \in L_{f}$, in which case $d$ is a local horizon direction that is retractive.

(2) $d \notin L_{f}$, in which case

$$
\lim _{\alpha \rightarrow \infty} \frac{f(x+\alpha d)}{\alpha}<0, \quad \forall x \in \mathbb{R}^{n} .
$$

In the latter case, we have $\lim _{\alpha \rightarrow \infty} f(x+\alpha d)=-\infty$ for all $x \in \operatorname{dom}(f)$, implying that $x+\alpha d \in \cap_{k=0}^{\infty} S_{k}$, for all sufficiently large $\alpha$. Thus, $d$ is a horizon direction with respect to $\operatorname{dom}(f)$.

Also, if there exists a direction $d$ with $d \in R_{f}$ but $d \notin L_{f}$, then by the preceding argument, we must have inf $\operatorname{s\in \mathbb {R}}^{n} f(x)=-\infty$, so that $\cap_{k=0}^{\infty} S_{k} \neq \emptyset$. If on the other hand, 
we have $R_{f}=L_{f}$, then by Proposition 6(a), $f$ attains its minimum over $\mathbb{R}^{n}$, so again $\cap_{k=0}^{\infty} S_{k} \neq \emptyset$.

In conclusion, if $f$ is bidirectionally flat, every asymptotic direction of $\left\{S_{k}\right\}$ is either a horizon direction with respect to $\operatorname{dom}(f)$, or else it is a local horizon direction that is retractive. Furthermore, $\cap_{k=0}^{\infty} S_{k} \neq \emptyset$.

As a special case of the preceding example, consider a set sequence $\left\{S_{k}\right\}$ defined by convex quadratic inequalities:

$$
S_{k}=\left\{x \mid x^{\prime} Q x+c^{\prime} x+b \leq \gamma_{k}\right\}
$$

For each asymptotic direction $d$, there are two possibilities:

(a) $d$ is a global horizon direction that satisfies $Q d=0$ and $c^{\prime} d<0$.

(b) $d$ is a local horizon direction that is also a lineality direction (satisfies $Q d=0$ and $c^{\prime} d=0$ ), and hence it is retractive.

Note that this property is not shared by nonconvex quadratic inequalities (unless the quadratic is strictly concave, see Example 2). As an example, for the subset of the plane

$$
S=\left\{\left(x_{1}, x_{2}\right) \mid x_{1} x_{2} \geq 1\right\},
$$

the asymptotic direction $(0,1)$ is not a local horizon direction [moving from $(-1,-1) \in$ $S$ along $(0,1)$ exits $S]$. It is also not retractive because, for some of its corresponding asymptotic sequences [e.g. the sequence $\{(1 / k, k)\}$ ], the requirement for retractiveness is not fulfilled.

\subsection{Critical directions}

We now introduce another type of asymptotic direction whose character reflects some of the root causes of emptiness of set intersections. The idea is that if two nested sequences $\left\{S_{k}^{1}\right\}$ and $\left\{S_{k}^{2}\right\}$, each with nonempty intersection by itself $\left(\cap_{k=0}^{\infty} S_{k}^{1} \neq \emptyset\right.$ and $\left.\cap_{k=0}^{\infty} S_{k}^{2} \neq \emptyset\right)$, and with nonempty intersection with the other $\left(S_{k}^{1} \cap S_{k}^{2} \neq \emptyset\right.$, for all $\left.k\right)$, are combined to form an empty intersection $\cap_{k=0}^{\infty}\left(S_{k}^{1} \cap S_{k}^{2}\right)$, then some of their common asymptotic directions must be "critical" in some sense. The following definition formulates this idea, and is motivated by properties of the asymptotic directions of level sets of bidirectionally flat functions.

Definition 4. Given a nested closed set sequence $\left\{S_{k}\right\}$ with nonempty intersection, we say that an asymptotic direction $d$ of $\left\{S_{k}\right\}$ is a critical direction with respect to a set $G$ if $d$ is neither a horizon direction of $\left\{S_{k}\right\}$ with respect to $G$, nor a retractive local horizon direction of $\left\{S_{k}\right\}$. An asymptotic direction of $\left\{S_{k}\right\}$ is referred to as noncritical with respect to $G$ if it is not critical with respect to $G$.

By convention, every asymptotic direction of $\left\{S_{k}\right\}$ is noncritical with respect to the empty set. In fact, Definitions 3 and 4 allow the possibility that the set $G$ is empty, and are consistent with this convention.

The definition of a critical and noncritical direction of a set sequence specializes naturally to the case of a single closed set $S$ by viewing the set as the constant sequence 
of sets $\left\{S_{k}\right\}$, where $S_{k}=S$ for all $k$; see Fig. 4. Note that all global horizon directions and all retractive local horizon directions are noncritical with respect to any nonempty set; see Fig. 5. Note also that all asymptotic directions of sequences of level sets of bidirectionally flat functions $f$, such as the ones considered in Example 4, are noncritical with respect to $\operatorname{dom}(f)$. In particular, sequences of nonempty convex quadratic level sets $S_{k}=\left\{x \mid x^{\prime} Q x+c^{\prime} x+b \leq \gamma_{k}\right\}$, where $\gamma_{k} \downarrow 0$, have noncritical asymptotic directions with respect to $\mathbb{R}^{n}$.

The significance of critical directions is illustrated by the following proposition, which shows that a sequence $\left\{S_{k}^{1} \cap \cdots \cap S_{k}^{r}\right\}$ with empty intersection must have an asymptotic direction that is critical for at least one of its components $\left\{S_{k}^{j}\right\}$ with respect to some of the other components. In particular, this implies that if all the components $\left\{S_{k}^{j}\right\}$ have no critical directions, then the intersection $\cap_{k=0}^{\infty}\left(S_{k}^{1} \cap \cdots \cap S_{k}^{r}\right)$ is nonempty. The following proposition is actually a special case of a more general proposition that we will prove shortly. We state the proposition separately because it is simpler and is still sufficient to show most of the results on existence of optimal solutions to be given in Section 4.

Proposition 7. Consider a set sequence $\left\{S_{k}\right\}$ of the form

$$
S_{k}=S_{k}^{1} \cap S_{k}^{2} \cap \cdots \cap S_{k}^{r}
$$

where $\left\{S_{k}^{j}\right\}, j=1, \ldots, r$, are nested sequences of nonempty closed sets such that $S_{k} \neq \emptyset$ for all $k$, and $\cap_{k=0}^{\infty} S_{k}^{j} \neq \emptyset$ for all $j$. If $\cap_{k=0}^{\infty} S_{k}=\emptyset$, there exists a nonempty index subset $J \subset\{1, \ldots, r\}$ such that $\cap_{k=0}^{\infty}\left(\cap_{j \in J} S_{k}^{j}\right)=\emptyset$, and an asymptotic direction of $\left\{\cap_{j \in J} S_{k}^{j}\right\}$ that for some $\bar{j} \in J$, is a critical direction of $\left\{S_{k}^{\bar{j}}\right\}$ with respect to $\cup_{j \in J-\{\bar{j}\}} \cap_{k=0}^{\infty} S_{k}^{j}$.

The proof of the preceding proposition will be obtained as a special case of the subsequent Proposition 8. Note a special case of the proposition: the intersection $\cap_{k=0}^{\infty} S_{k}$ is nonempty if the sets $S_{k}, k=0,1, \ldots$, and $\cap_{k=0}^{\infty} S_{k}^{j}, j=1, \ldots, r$, are nonempty, and the asymptotic directions of each sequence $\left\{S_{k}^{j}\right\}$ are noncritical with respect to $\mathbb{R}^{n}$. The conclusion of the proposition cannot be replaced by the stronger conclusion that if $\cap_{k=0}^{\infty} S_{k}=\emptyset$, there exists an asymptotic direction of $\left\{S_{k}\right\}$ that for some $\bar{j} \in\{1, \ldots, r\}$, is a critical direction of $\left\{S_{k}^{\bar{j}}\right\}$ with respect to $\cup_{j \in\{1, \ldots, r\}-\{\bar{j}\}} \cap_{k=0}^{\infty} S_{k}^{j}$. This is shown by the following counterexample.

Example 5. (Counterexample) Consider the following three sequences of nonempty closed sets:

$$
\begin{aligned}
& S_{k}^{1}=\{(x, y, z) \mid 0 \leq z \leq 1 / k\}, \quad k=0,1, \ldots, \\
& S_{k}^{2}=\{(x, y, z) \mid x>0,1 / x \leq z\}, \quad k=0,1, \ldots, \\
& S_{k}^{3}=\left\{(x, y, z) \mid y \geq x^{2}\right\}, \quad k=0,1, \ldots,
\end{aligned}
$$

Each sequence has a nonempty intersection, and $S_{k}=S_{k}^{1} \cap S_{k}^{2} \cap S_{k}^{3}$ is nonempty for $k=0,1, \ldots$. The intersection of $\cap_{k=0}^{\infty} S_{k}^{1}$ and $\cap_{k=0}^{\infty} S_{k}^{2}$ is empty, and hence $\cap_{k=0}^{\infty} S_{k}$ is 


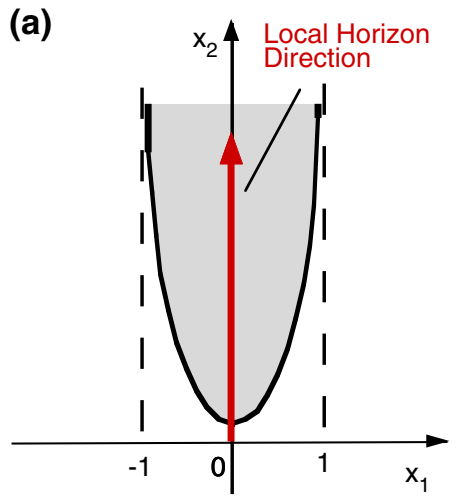

(c)

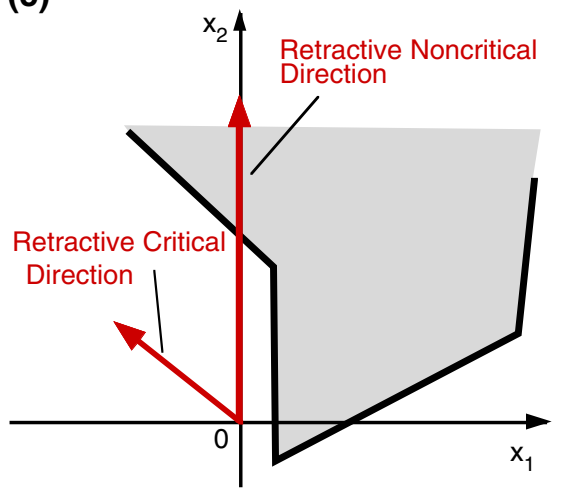

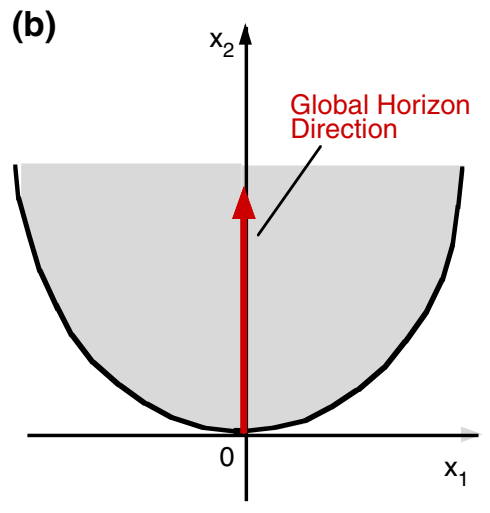

(d)

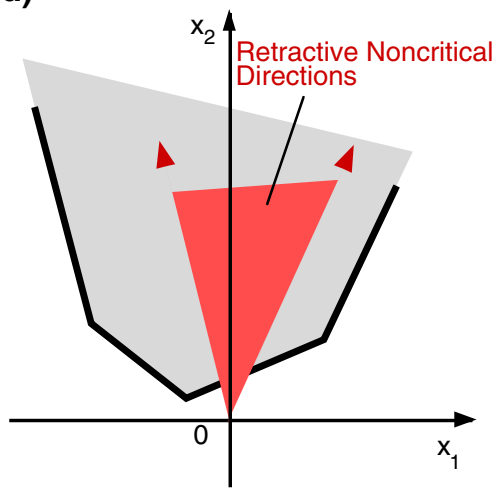

Fig. 4 Examples of horizon, critical, and noncritical directions of various sets. In (a), the asymptotic direction $(0, \beta), \beta>0$, is a horizon direction and a noncritical direction with respect to any subset of the set $\left\{\left(x_{1}, x_{2}\right) \mid-1<x_{1}<1\right\}$. It is not a horizon direction and it is a critical direction with respect to any other subset. In (b), the given set is $\left\{\left(x_{1}, x_{2}\right) \mid x_{1}^{2} \leq x_{2}\right\}$, and all its asymptotic directions $[(0, \beta), \beta>0]$ are global horizon directions and hence noncritical directions with respect to any subset. The sets in (c) and (d) are retractive. In (c), some asymptotic directions [such as $(0, \beta), \beta>0$ ] are retractive local horizon directions, while others are not and are therefore critical with respect to some sets. In (d), all asymptotic directions are retractive local horizon directions and are therefore noncritical with respect to any set

also empty. However, all asymptotic directions of $\left\{S_{k}\right\}$ are along the $y$-axis, and are global horizon directions of $\left\{S_{k}^{3}\right\}$. These directions are also retractive local horizon directions of $\left\{S_{k}^{1}\right\}$ and $\left\{S_{k}^{2}\right\}$. Hence they are noncritical directions of $\left\{S_{k}^{\bar{j}}\right\}$ with respect to $\mathbb{R}^{3}$ (and hence also with respect to the smaller set $\cup_{j \in\{1,2,3\}-\{\bar{j}\}} \cap_{k=0}^{\infty} S_{k}^{j}$ ) for all $\bar{j} \in\{1,2,3\}$.

As an illustration of how Proposition 7 may be applied, consider a sequence $\left\{S_{k}\right\}$ of the form

$$
S_{k}=X \cap S_{k}^{1} \cap S_{k}^{2} \cap \cdots \cap S_{k}^{r},
$$




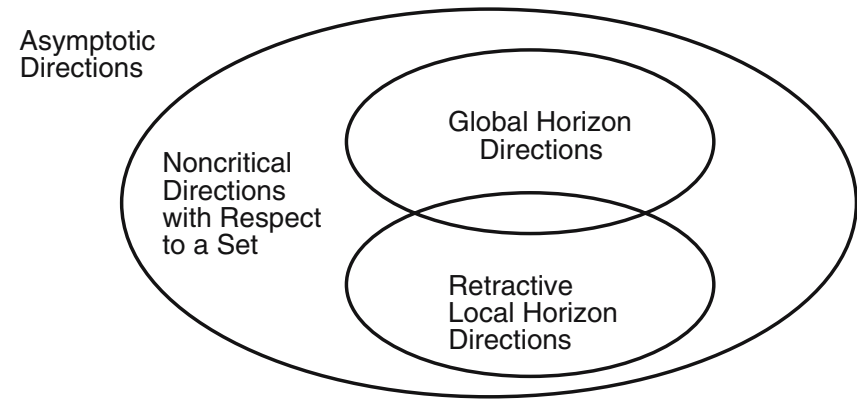

Fig. 5 Relations between different types of asymptotic directions

where for each $j, S_{k}^{j}$ is given by

$$
S_{k}^{j}=\left\{x \mid x^{\prime} Q_{j} x+c_{j}^{\prime} x+b_{j} \leq \gamma_{k}^{j}\right\},
$$

and $Q_{j}$ is a real symmetric positive semidefinite matrix, $c_{j}$ is a vector, $b_{j}$ is a scalar, and $\left\{\gamma_{k}^{j}\right\}$ is a scalar positive sequence with $\gamma_{k}^{j} \downarrow 0$. Furthermore, $X$ is a nonempty closed set such that all its asymptotic directions are either global horizon directions or retractive local horizon directions. Then, all the asymptotic directions of $\left\{S_{k}^{j}\right\}$ and $X$ are noncritical with respect to $\mathbb{R}^{n}$, Proposition 7 applies, and shows that $\cap_{k=0}^{\infty} S_{k}$ is nonempty, assuming the sets $S_{k}$ are nonempty and $\cap_{k=0}^{\infty} S_{k}^{j}=\left\{x \mid x^{\prime} Q_{j} x+c_{j}^{\prime} x+b_{j} \leq 0\right\}$ is nonempty for all $j$. This result generalizes a theorem of Luo and Zhang (see [21], Theorem 1) for the purely quadratic case $\left(X=\mathbb{R}^{n}\right)$.

The reasoning used above applies also in the more general case where the convex quadratic functions are replaced by any real-valued bidirectionally flat functions. However, Proposition 7 does not apply to set intersections involving extended real-valued bidirectionally flat functions (unless the domains of these functions are identical). The following proposition is a generalization of Proposition 7, which will allow us to deal with such situations (see Proposition 9 later in this section). Its proof uses Proposition 1 and a nontrivial generalization of an induction argument from [21].

Proposition 8. Consider a set sequence $\left\{S_{k}\right\}$ of the form

$$
S_{k}=X_{k} \cap S_{k}^{1} \cap S_{k}^{2} \cap \cdots \cap S_{k}^{r},
$$

where $\left\{X_{k}\right\}$ and $\left\{S_{k}^{j}\right\}, j=1, \ldots, r$, are nested sequences of nonempty closed sets, such that $S_{k} \neq \emptyset$ for all $k, \cap_{k=0}^{\infty} X_{k} \neq \emptyset$, and $\cap_{k=0}^{\infty} S_{k}^{j} \neq \emptyset$ for all $j$. If $\cap_{k=0}^{\infty} S_{k}=\emptyset$, there exists a nonempty index subset $J \subset\{1, \ldots, r\}$ such that $\cap_{k=0}^{\infty}\left(X_{k} \cap\left(\cap_{j \in J} S_{k}^{j}\right)\right)=\emptyset$, and an asymptotic direction d of $\left\{X_{k} \cap\left(\cap_{j \in J} S_{k}^{j}\right)\right\}$ such that at least one of the following two holds:

(1) $d$ is not a retractive local horizon direction of $\left\{X_{k}\right\}$.

(2) For some $\bar{j} \in J, d$ is a critical direction of $\left\{S_{k}^{\bar{j}}\right\}$ with respect to the $\operatorname{set} \cup_{j \in J-\{\bar{j}\}} \cap_{k=0}^{\infty}$ $\left(X_{k} \cap S_{k}^{j}\right.$ ) (with the convention that this set equals $\cap_{k=0}^{\infty} X_{k}$ when $J=\{\bar{j}\}$ ). 
Proof. We assume the contrary, i.e. that for every $J \subset\{1, \ldots, r\}$ such that $\cap_{k=0}^{\infty}\left(X_{k} \cap\left(\cap_{j \in J} S_{k}^{j}\right)\right)=\varnothing$, all asymptotic directions of $\left\{X_{k} \cap\left(\cap_{j \in J} S_{k}^{j}\right)\right\}$ are noncritical directions of each $\left\{S_{k}^{\bar{j}}\right\}, \bar{j} \in J$, with respect to $\cup_{j \in J-\{\bar{j}\}} \cap_{k=0}^{\infty}\left(X_{k} \cap S_{k}^{j}\right)$ (which by our convention equals $\cap_{k=0}^{\infty} X_{k}$ when $r=1$ ), while they are also retractive local horizon directions of $\left\{X_{k}\right\}$.

Let $A$ be the set of asymptotic directions of $\left\{S_{k}\right\}$ (which is nonempty since $\cap_{k=0}^{\infty}$ $\left.S_{k}=\emptyset\right)$. Then, taking $J=\{1, \ldots, r\}$, we see that there must exist some $j_{1} \in\{1, \ldots, r\}$ and some $d \in A$ that is a horizon direction of $\left\{S_{k}^{j_{1}}\right\}$ with respect to $\cup_{j \in J-\left\{j_{1}\right\}} \cap_{k=0}^{\infty}$ $\left(X_{k} \cap S_{k}^{j}\right)$; otherwise each $d \in A$ would be retractive for $\left\{X_{k}\right\}$ and for all $\left\{S_{k}^{j}\right\}$, and hence also retractive for $\left\{S_{k}\right\}$, so, by Proposition 1, the hypothesis $\cap_{k=0}^{\infty} S_{k}=\emptyset$ would be contradicted.

Consider the sequence $\left\{S_{k}(1)\right\}$, obtained from $\left\{S_{k}\right\}$ when the sets $S_{k}^{j_{1}}$ are eliminated, i.e.

$$
S_{k}(1)=X_{k} \cap\left(\cap_{j \in J-\left\{j_{1}\right\}} S_{k}^{j}\right)
$$

We argue by contradiction that $\cap_{k=0}^{\infty} S_{k}(1)=\emptyset$. Suppose that this is not so. Take any $x \in \cap_{k=0}^{\infty} S_{k}(1)$, and consider the direction $d \in A$ that is a horizon direction of $\left\{S_{k}^{j_{1}}\right\}$ with respect to $\cup_{j \in J-\left\{j_{1}\right\}} \cap_{k=0}^{\infty}\left(X_{k} \cap S_{k}^{j}\right)$. Since $d \in A, d$ is also a retractive local horizon direction of $\left\{X_{k}\right\}$. Then we have

$$
\begin{array}{ll}
x+\alpha d \in \cap_{k=0}^{\infty} X_{k}, & \forall \alpha \text { sufficiently large, } \\
x+\alpha d \in \cap_{k=0}^{\infty} S_{k}^{j_{1}}, & \forall \alpha \text { sufficiently large. }
\end{array}
$$

For the case where $J$ has cardinality 2 or more, we show below that, for all $\bar{j} \in J-\left\{j_{1}\right\}$, we also have

$$
x+\alpha d \in \cap_{k=0}^{\infty} S_{k}^{\bar{j}}, \quad \forall \alpha \text { sufficiently large, }
$$

which, combined with Eqs. (5) and (6), contradicts the assumed emptiness of $\cap_{k=0}^{\infty} S_{k}$.

Indeed, for any $\bar{j} \in J-\left\{j_{1}\right\}$, under our working hypothesis, there are two possibilities:

1. $d$ is a retractive local horizon direction of $\left\{S_{k}^{\bar{j}}\right\}$, in which case, since $x \in \cap_{k=0}^{\infty} S_{k}^{\bar{j}}$, Eq. (7) holds.

2. $d$ is a horizon direction of $\left\{S_{k}^{\bar{j}}\right\}$ with respect to $\cup_{j \in J-\{\bar{j}\}} \cap \cap_{k=0}^{\infty}\left(X_{k} \cap S_{k}^{j}\right)$, and since $j_{1} \in J-\{\bar{j}\}, d$ is a horizon direction of $\left\{S_{k}^{\bar{j}}\right\}$ with respect to $\cap_{k=0}^{\infty}\left(X_{k} \cap S_{k}^{j_{1}}\right)$. Since, by Eqs. (5) and (6),

$$
x+\alpha d \in \cap_{k=0}^{\infty}\left(X_{k} \cap S_{k}^{j_{1}}\right)
$$

for all $\alpha$ sufficiently large, this further implies that

$$
(x+\alpha d)+\bar{\alpha} d \in \cap_{k=0}^{\infty} S_{k}^{\bar{j}}
$$

for all $\bar{\alpha}$ sufficiently large. Thus, Eq. (7) holds in this case as well. 
In conclusion, Eq. (7) holds for all $\bar{j} \in J-\left\{j_{1}\right\}$. Combining Eqs. (5)-(7), we see that $x+\alpha d \in \cap_{k=0}^{\infty} S_{k}$ for sufficiently large $\alpha$. Thus, the contradiction argument showing that $\cap_{k=0}^{\infty} S_{k}(1)=\emptyset$ is complete.

We may now repeat this argument, with $S_{k}$ replaced by $S_{k}(1)$ and $J$ redefined as

$$
J=\{1, \ldots, r\}-\left\{j_{1}\right\}
$$

to obtain another index $j_{2} \neq j_{1}$ such that $\cap_{k=0}^{\infty} S_{k}(2)=\emptyset$, where $S_{k}(2)$ is the set formed by intersection of all the sets $S_{k}^{j}$ except $S_{k}^{j_{1}}$ and $S_{k}^{j_{2}}$, i.e.

$$
S_{k}(2)=X_{k} \cap\left(\cap_{j \in J-\left\{j_{2}\right\}} S_{k}^{j}\right) \text {. }
$$

Continuing the process, after $r$ steps we conclude that $\cap_{k=0}^{\infty} S_{k}(r)=\cap_{k=0}^{\infty} X_{k}=\emptyset$, which contradicts the hypothesis.

Note that Proposition 7 is obtained as the special case of Proposition 8 where the sets $X_{k}$ are all equal to $\mathbb{R}^{n}$. By combining Example 4 with the preceding proposition, we obtain a generalization of a result given as Exercise 2.7 of [11] (the result of this exercise is the special case where $X=\mathbb{R}^{n}$ in the following proposition).

Proposition 9. Consider a set sequence $\left\{S_{k}\right\}$ of the form

$$
S_{k}=X_{k} \cap S^{1} \cap S^{2} \cap \cdots \cap S^{r},
$$

where

$$
X_{k}=X \cap S_{k}^{0}, \quad k=0,1, \ldots,
$$

and $X$ is a closed set such that all its asymptotic directions are retractive local horizon directions. Furthermore, $S_{k}^{0}$ and $S^{j}, j=1, \ldots, r$, are given by

$$
S_{k}^{0}=\left\{x \mid f_{0}(x) \leq \gamma_{k}\right\}, \quad k=0,1, \ldots, \quad S^{j}=\left\{x \mid f_{j}(x) \leq 0\right\}, \quad j=1, \ldots, r,
$$

where $\left\{\gamma_{k}\right\}$ is a scalar nonnegative sequence with $\gamma_{k} \downarrow 0$, and for each $j=0,1, \ldots, r$, $f_{j}: \mathbb{R}^{n} \mapsto(-\infty, \infty]$ is a closed proper convex function that is bidirectionally flat. Assume that $S_{k}$ is nonempty for all $k$, and that $\cap_{k=0}^{\infty} X_{k} \subset \cap_{j=1}^{r} \operatorname{dom}\left(f_{j}\right)$. Then $\cap_{k=0}^{\infty} S_{k}$ is nonempty.

Proof. We first show that $\cap_{k=0}^{\infty} X_{k} \neq \emptyset$. Let $A$ be the set of asymptotic directions of $\left\{X_{k}\right\}$. Then for all $d \in A$, we have $x+\alpha d \in X$ for all $x \in X$ and $\alpha$ sufficiently large, since the asymptotic directions of $X$ are local horizon directions. Also, $d \in R_{f_{0}}$. There are two cases (see Example 4):

(1) $d \notin L_{f_{0}}$ for some $d \in A$. Then, since $f_{0}$ is closed, convex, and bidirectionally flat, $\lim _{\alpha \rightarrow \infty} f_{0}(x+\alpha d)=-\infty$ for all $x \in \operatorname{dom}\left(f_{0}\right)$, so that $x+\alpha d \in \cap_{k=0}^{\infty} X_{k}$ for all $x \in X \cap \operatorname{dom}\left(f_{0}\right)$ and all $\alpha$ sufficiently large. It follows that $\cap_{k=0}^{\infty} X_{k} \neq \emptyset$.

(2) $d \in L_{f_{0}}$ for all $d \in A$. Then, since $f_{0}$ is closed, convex, and bidirectionally flat, all $d \in A$ are retractive for $\left\{S_{k}^{0}\right\}$ as well as retractive for $X$. Hence, all $d \in A$ are retractive for $\left\{X_{k}\right\}$, and it follows that $\cap_{k=0}^{\infty} X_{k} \neq \varnothing$ by Proposition 1 . 
We will now prove that $\cap_{k=0}^{\infty} S_{k} \neq \varnothing$ by contradiction. In particular, we assume that $\cap_{k=0}^{\infty} S_{k}=\emptyset$, and we will verify that the conclusion of Proposition 8 does not hold. Indeed, consider an index subset $J \subset\{1, \ldots, r\}$ such that $\cap_{k=0}^{\infty}\left(X_{k} \cap\left(\cap_{j \in J} S_{k}^{j}\right)\right)=\emptyset$, and let $d$ be an asymptotic direction of $\left\{X_{k} \cap\left(\cap_{j \in J} S_{k}^{j}\right)\right\}$. We will show that $d$ is a noncritical direction of all $\left\{S_{k}^{\bar{j}}\right\}, \bar{j} \in J$, with respect to $\cap_{k=0}^{\infty} X_{k}$ [and hence also with respect to the smaller set $\left.\cup_{j \in J-\{\bar{j}\}} \cap_{k=0}^{\infty}\left(X_{k} \cap S_{k}^{j}\right)\right]$, while it is a retractive local horizon direction of $\left\{X_{k}\right\}$, thereby contradicting the conclusion of Proposition 8 .

We first note that $d \in R_{f_{j}}$ for all $j \in J$. It follows that for each $j \in J$, either $d \in L_{f_{j}}$, in which case $d$ is a horizon direction of $S^{j}$ that is retractive, or $d \notin L_{f_{j}}$, in which case (since $f_{j}$ is closed, convex, and bidirectionally flat) $d$ is a horizon direction of $S^{j}$ with respect to $\operatorname{dom}\left(f_{j}\right)$, and hence also a horizon direction of $S^{j}$ with respect to $\cap_{k=0}^{\infty} X_{k}$ [since $\cap_{k=0}^{\infty} X_{k} \subset \operatorname{dom}\left(f_{j}\right)$ by assumption]. Thus, $d$ is a noncritical direction of all $S^{j}, j \in J$, with respect to $\cap_{k=0}^{\infty} X_{k}$.

We also have that $d \in R_{f_{0}}$. Assume that $d \notin L_{f_{0}}$, and let $x$ be any vector in $X \cap S_{0}^{0} \cap S^{1} \cap \cdots \cap S^{r}$. Then for all $\alpha$ sufficiently large, we have

$$
x+\alpha d \in \bigcap_{k=0}^{\infty} S_{k}^{0}
$$

since $x \in \operatorname{dom}\left(f_{0}\right)$ and $f_{0}$ is closed, convex, and bidirectionally flat, so that $\lim _{\alpha \rightarrow \infty} f_{0}(x+\alpha d)=-\infty$. Furthermore, for all $\alpha$ sufficiently large, we have

$$
x+\alpha d \in X \cap\left(\cap_{j \in J} S^{j}\right),
$$

since $d$ is a local horizon direction of $X$ and a direction of recession of each $S^{j}, j \in J$. Equations (8) and (9) contradict the assumed emptiness of the intersection of $\left\{X_{k} \cap\left(\cap_{j \in J} S^{j}\right)\right\}$. Hence $d \in L_{f_{0}}$, from which by arguing as in case (2) above, we see that $d$ is a retractive local horizon direction of $\left\{X_{k}\right\}$. Thus the conclusion of Proposition 8 is contradicted, and it follows that $\cap_{k=0}^{\infty} S_{k}$ is nonempty.

Example 6. (A Counterexample for Bidirectionally Flat Functions) To see that the assumption $\cap_{k=0}^{\infty} X_{k} \subset \cap_{j=1}^{r} \operatorname{dom}\left(f_{j}\right)$ is essential in Proposition 9, let $X=\mathbb{R}^{2}$ and consider the following two bidirectionally flat functions $f_{0}$ and $f_{1}$ defined on $\mathbb{R}^{2}$ :

$$
f_{0}\left(x_{1}, x_{2}\right)=x_{1}, \quad f_{1}\left(x_{1}, x_{2}\right)=\phi\left(x_{1}\right)-x_{2},
$$

where the function $\phi: \mathbb{R} \mapsto(-\infty, \infty]$ is convex, closed, and coercive with $\operatorname{dom}(\phi)=$ $(0,1)$ [e.g. $\phi(t)=-\ln t-\ln (1-t)$ for $0<t<1]$. Take also $\left\{\gamma_{k}\right\}$ to be any sequence in $(0,1)$ with $\gamma_{k} \downarrow 0$, so

$$
S_{k}=\left\{x \mid x_{1} \leq \gamma_{k}, \phi\left(x_{1}\right)-x_{2} \leq 0\right\} .
$$

Then, it can be verified that $S_{k} \neq \varnothing$ for every $k$ [take $x_{1} \downarrow 0$ and $x_{2} \geq \phi\left(x_{1}\right)$ ], and we have

$$
\bigcap_{k=0}^{\infty} X_{k}=\bigcap_{k=0}^{\infty} S_{k}^{0}=\left\{x \mid f_{0}(x) \leq 0\right\}=\left\{x \mid x_{1} \leq 0, x_{2} \in \mathbb{R}\right\}
$$




$$
\bigcap_{k=0}^{\infty} S_{k}^{1}=\left\{x \mid f_{1}(x) \leq 0\right\}=\left\{x \mid 0<x_{1}<1, x_{2} \in \mathbb{R}\right\}=\operatorname{dom}\left(f_{1}\right) .
$$

The two sets are disjoint, so the conclusion of Proposition 9 is violated, and in particular we have

$$
\bigcap_{k=0}^{\infty} S_{k}=\left\{x \mid f_{0}(x) \leq 0, f_{1}(x) \leq 0\right\}=\left(\bigcap_{k=0}^{\infty} X_{k}\right) \cap\left(\bigcap_{k=0}^{\infty} S_{k}^{1}\right)=\emptyset .
$$

We now consider sets defined by a finite number of concave quadratic inequalities. We have seen that the asymptotic directions of such sets are retractive (see Proposition 4). We will delineate circumstances under which the asymptotic directions are also local horizon directions, so that they are noncritical.

Example 7. (Level sets of concave quadratic functions) Consider a set of the form

$$
S=\left\{x \mid x^{\prime} Q x+c^{\prime} x+b \geq 0\right\},
$$

where $Q$ is a positive semidefinite $n \times n$ matrix, $c$ is a vector in $\mathbb{R}^{n}$, and $b$ is a scalar. We first derive the set of asymptotic directions of $S$. We consider two cases:

(a) $Q=0$. Then $A_{S}$ is the set of nonzero directions of recession of the convex function $-\left(c^{\prime} x+b\right)$, as discussed earlier:

$$
A_{S}=\left\{d \mid c^{\prime} d \geq 0, d \neq 0\right\} .
$$

(b) $Q \neq 0$. Then we claim that the asymptotic directions of $S$ are the nonzero vectors in $\mathbb{R}^{n}$ :

$$
A_{S}=\{d \mid d \neq 0\} .
$$

Indeed, take any nonzero vector $d$ and any $y$ such that

$$
y^{\prime} Q y+c^{\prime} d>0 .
$$

We will show that for sufficiently large $k$, the sequence of vectors

$$
x_{k}=k d+\sqrt{k} y
$$

is an asymptotic sequence of $S$ that corresponds to $d$. We note that $\left\|x_{k}\right\| \rightarrow \infty$ and that $x_{k} /\left\|x_{k}\right\| \rightarrow d /\|d\|$. Furthermore, we have

$$
x_{k}^{\prime} Q x_{k}+c^{\prime} x_{k}+b=k^{2} d^{\prime} Q d+2 k \sqrt{k} y^{\prime} Q d+k y^{\prime} Q y+k c^{\prime} d+\sqrt{k} c^{\prime} y+b .
$$

If $d^{\prime} Q d>0$, clearly we have $x_{k} \in S$ for sufficiently large $k$. On the other hand, if $d^{\prime} Q d=0$ (equivalently, $Q d=0$, since $Q$ is positive semidefinite), then from Eq. (10),

$$
x_{k}^{\prime} Q x_{k}+c^{\prime} x_{k}+b=k\left(y^{\prime} Q y+c^{\prime} d\right)+\sqrt{k} c^{\prime} y+b .
$$

Since $y^{\prime} Q y+c^{\prime} d>0$, we again have $x_{k} \in S$ for sufficiently large $k$. Thus, for some integer $\bar{k}$, the subsequence $\left\{x_{k} \mid k \geq \bar{k}\right\}$ fulfills the requirements for an asymptotic sequence of $S$ corresponding to $d$, and it follows that $d$ is an asymptotic direction. 
We know from Proposition 4 that all asymptotic directions of $S$ are retractive. However, some of these directions may be critical because they are not local horizon directions. For example, let

$$
S=\left\{\left(x_{1}, x_{2}\right) \mid x_{1} \leq x_{2}^{2}\right\}
$$

Then the vector $(1,0)$ is a retractive asymptotic direction (by the preceding analysis), but is not a local horizon direction. More generally, the set of asymptotic directions $S$ that are not local horizon directions is the set

$$
\bar{A}=\left\{d \mid Q d=0, \quad c^{\prime} d<0, \quad d \neq 0\right\} .
$$

To see this, note that it is true in the case where $Q=0$, where $A_{S}=\{d \mid d \neq 0\}$, and $\bar{A}=\emptyset$. In the case where $Q \neq 0$, note that for any $x \in S, d \in A_{S}$, and $\alpha \geq 0$, we have $(x+\alpha d)^{\prime} Q(x+\alpha d)+c^{\prime}(x+\alpha d)+b=x^{\prime} Q x+c^{\prime} x+b+\alpha^{2} d^{\prime} Q d+\alpha(2 Q x+c)^{\prime} d$.

It follows that $x+\alpha d \in S$ for sufficiently large $\alpha$ if and only if either $d^{\prime} Q d>0$, or $Q d=0$ and $c^{\prime} d \geq 0$. This proves Eq. (11).

Consider now a set sequence $\left\{S_{k}\right\}$ defined by a finite number of concave quadratic inequalities:

$$
S_{k}=P \cap\left\{x \mid x^{\prime} Q_{j} x+c_{j}^{\prime} x+b_{j} \geq \gamma_{k}^{j}, j=1, \ldots, r\right\},
$$

where $\left\{\gamma_{k}^{j}\right\}$ are scalar sequences with $\gamma_{k}^{j} \uparrow 0, P$ is a polyhedral set, $Q_{j}$ are nonzero positive semidefinite $n \times n$ matrices, $c_{j}$ are vectors in $\mathbb{R}^{n}$, and $b_{j}$ are scalars. A slight extension of the preceding analysis shows that the asymptotic directions of $\left\{S_{k}\right\}$ form a subset of the nonzero vectors in the recession cone $R_{P}$, and all of them are retractive. A sufficient condition for all asymptotic directions to be noncritical local horizon directions of $\left\{S_{k}\right\}$ is that $R_{P} \cap N\left(Q_{j}\right)=\{0\}$ for all $j=1, \ldots, r$, where $N\left(Q_{j}\right)$ is the nullspace of $Q_{j}$. This is true in particular if all the matrices $Q_{j}$ are positive definite.

Let us also introduce a class of nonconvex functions whose level sets have the essential property needed for application of Propositions 7 and 8 , so that they can be used in place of convex quadratic or real-valued bidirectionally flat functions to assert nonemptiness of a set intersection.

Example 8. Let $\left\{S_{k}\right\}$ be a level set sequence

$$
S_{k}=\left\{x \mid f(x) \leq \gamma_{k}\right\},
$$

defined by a function $f$ of the form Eq. (3), where $A$ is an $m \times n$ matrix, $c$ is a vector, $b$ is a scalar, and $h: \mathbb{R}^{m} \mapsto \mathbb{R}$ is a closed function satisfying Eq. (4). We assume that $\left\{\gamma_{k}\right\}$ is a scalar positive sequence with $\gamma_{k} \downarrow 0$, and that $\{x \mid f(x) \leq 0\}=\cap_{k=0}^{\infty} S_{k}$ is nonempty.

We have

$$
f(x+\alpha d)=h(A x+\alpha A d)+c^{\prime}(x+\alpha d)+b .
$$


A nonzero vector $d$ is a local horizon direction if and only if for every $x$ with $f(x) \leq 0$, there exists $\bar{\alpha} \geq 0$ such that for all $\alpha \geq \bar{\alpha}$, we have $f(x+\alpha d) \leq 0$. In view of the coercivity property of $h$, this is true if and only if $A d=0$ and $c^{\prime} d \leq 0$. A nonzero vector $d$ is a global horizon direction if and only if, for every $x \in \mathbb{R}^{n}$, there exists $\bar{\alpha} \geq 0$ such that for all $\alpha \geq \bar{\alpha}$, we have $f(x+\alpha d) \leq 0$. This is true if and only if $A d=0$ and $c^{\prime} d<0$. Thus, every asymptotic direction of $\left\{S_{k}\right\}$ is either a global horizon direction, or else it is a local horizon direction that is retractive, i.e. it is noncritical with respect to $\mathbb{R}^{n}$.

Thus, if we have functions $f_{1}, \ldots, f_{r}$, each of the form $f$ above, and $\left\{x \mid f_{j}(x) \leq 0\right\}$ is nonempty for $j=1, \ldots, r$, and $\left\{x \mid f_{1}(x) \leq \gamma_{k}, \ldots, f_{r}(x) \leq \gamma_{k}\right\}$ is nonempty for $k=0,1, \ldots$, then we can apply Proposition 7 [with $S_{k}^{j}=\left\{x \mid f_{j}(x) \leq \gamma_{k}\right\}$ ] to conclude that $\left\{x \mid f_{1}(x) \leq 0, \ldots, f_{r}(x) \leq 0\right\}$ is nonempty.

\section{Existence of optimal solutions}

We will now consider the problem of minimizing a closed function $f: \mathbb{R}^{n} \mapsto(-\infty, \infty]$ over a closed set $X \subset \mathbb{R}^{n}$. Let $\left\{\gamma_{k}\right\}$ be a scalar sequence with $\gamma_{k} \downarrow \inf _{x \in X} f(x)$, and consider the (nonempty) level sets

$$
V_{k}=\left\{x \mid f(x) \leq \gamma_{k}\right\}
$$

The set of vectors that minimize $f$ over $X$ is the intersection

$$
X^{*}=\cap_{k=0}^{\infty}\left(X \cap V_{k}\right),
$$

so to show existence of an optimal solution, we can use asymptotic directions, and the results of Sects. 2 and 3. In particular, it is sufficient to show that all asymptotic directions of the sequence $\left\{X \cap V_{k}\right\}$ are retractive (see Proposition 1). Also, if $X$ is polyhedral or more generally, if it is the vector sum of a compact set and a polyhedral cone $N$, it is sufficient to show that all the asymptotic directions of $\left\{V_{k}\right\}$ that belong to $N$ are retractive (see Proposition 2).

We first consider the case of a closed convex function $f: \mathbb{R}^{n} \mapsto(-\infty, \infty]$ that is quasiconvex, in the sense that all its level sets $\{x \mid f(x) \leq \gamma\}$ are convex. The preceding observation, together with Proposition 6(b), yields the following proposition (new to our knowledge). Notice that, in the case where $f$ is convex, $R_{\Gamma}$ and $L_{\Gamma}$ in the following proposition reduce to, respectively, the recession cone $R_{f}$ and constancy space $L_{f}$ of $f$.

Proposition 10 (Quasiconvex Cost Function). Let $f: \mathbb{R}^{n} \mapsto(-\infty, \infty]$ be a closed quasiconvex function, and let $X$ be a closed subset of $\mathbb{R}^{n}$ such that $X \cap \operatorname{dom}(f) \neq \emptyset$. Let $\Gamma$ be the set of all $\gamma>\inf _{x \in X} f(x)$, and denote

$$
R_{\Gamma}=\cap_{\gamma \in \Gamma} R_{\gamma}, \quad L_{\Gamma}=\cap_{\gamma \in \Gamma} L_{\gamma},
$$

where $R_{\gamma}$ and $L_{\gamma}$ are the recession cone and the lineality space of the level set $\{x \mid f(x)$ $\leq \gamma\}$, respectively. Then $f$ attains a minimum over $X$ under any one of the following two conditions: 
(1) $A_{X} \cap R_{\Gamma} \subset L_{X} \cap L_{\Gamma}$.

(2) $X$ is retractive and $A_{X} \cap R_{\Gamma} \subset L_{\Gamma}$.

Proof. Let

$$
V_{k}=\left\{x \mid f(x) \leq \gamma_{k}\right\},
$$

where $\left\{\gamma_{k}\right\}$ is a scalar sequence such that $\gamma_{k} \downarrow \inf _{x \in X} f(x)$. We show that under each of the two conditions, the intersection $\cap_{k=0}^{\infty}\left(X \cap V_{k}\right)$ (which is the set of minimizing points) is nonempty.

Let condition (1) hold. The sets $X \cap V_{k}$ are nonempty, closed, convex, and nested. Furthermore, they have the same set of asymptotic directions, $A_{X} \cap R_{\Gamma}$, and the same lineality space $L_{X} \cap L_{\Gamma}$, while by assumption, $A_{X} \cap R_{\Gamma} \subset L_{X} \cap L_{\Gamma}$. The result follows from Proposition 6(a).

Let condition (2) hold. The sets $V_{k}$ are nested and the intersection $X \cap V_{k}$ is nonempty for all $k$. Furthermore, the sets $V_{k}$ have the same recession cone, $R_{\Gamma}$, and the same lineality space, $L_{\Gamma}$, while by assumption, $A_{X} \cap R_{\Gamma} \subset L_{\Gamma}$. The result follows from Proposition 6(b).

We now give a result involving convex bidirectionally flat functions (see Example 4), which relies on the use of horizon directions. The following proposition extends an existence result given in [11] as Exercise 2.7(c), and also a result of Belousov [8], which dates to 1977 (as discussed in [10]) and deals with the case where the functions involved are convex polynomial functions (a special case of bidirectionally flat functions, as discussed in Example 4). The purely quadratic case of this result $\left(X=\mathbb{R}^{n}\right.$ and $f_{j}$ is convex quadratic) was independently given by Terlaky [28]; see also Luo and Zhang [21], who prove some additional results that involve in part nonconvex quadratic functions. A related existence result is given by Bank and Mandel [7] for quasiconvex polynomial functions whose level sets have common recession directions that are finitely generated, and with some of the variables constrained to be integer-valued (see [7], Theorem 7.4).

Proposition 11 (Bidirectionally Flat Functions). For $j=0,1, \ldots, r$, let $f_{j}: \mathbb{R}^{n} \mapsto$ $(-\infty, \infty]$ be closed proper convex functions that are bidirectionally flat, and let $X$ be a closed set such that all its asymptotic directions are retractive local horizon directions. Assume that $\left(X \cap \operatorname{dom}\left(f_{0}\right)\right) \subset \cap_{j=1}^{r} \operatorname{dom}\left(f_{j}\right)$. Then, the problem

$$
\begin{aligned}
& \text { minimize } f_{0}(x) \\
& \text { subject to } x \in X, \quad f_{j}(x) \leq 0, \quad j=1, \ldots, r,
\end{aligned}
$$

has at least one optimal solution if and only if its optimal value is finite.

Proof. Assume that $f^{*}$, the optimal value, is finite, and let $\left\{\gamma_{k}\right\}$ be a scalar sequence such that $\gamma_{k} \downarrow 0$. Consider the set sequence $\left\{S_{k}\right\}$ given by

$$
S_{k}=X_{k} \cap S^{1} \cap S^{2} \cap \cdots \cap S^{r}
$$

where

$$
\begin{aligned}
X_{k} & =X \cap S_{k}^{0}, \quad S_{k}^{0}=\left\{x \mid f_{0}(x)-f^{*} \leq \gamma_{k}\right\}, \quad k=0,1, \ldots, \\
S^{j} & =\left\{x \mid f_{j}(x) \leq 0\right\}, \quad j=1, \ldots, r .
\end{aligned}
$$

Using Proposition 9, we have that $\cap_{k=0}^{\infty} S_{k}$, the optimal solution set, is nonempty. 
We were informed by a referee that Belousov ([8], Theorem 14 of Chap. 13) proved an existence result for minimizing a convex polynomial function over a set $X$ which is the vector sum of a convex compact set and a polyhedral cone. It is readily seen that such a set satisfies the assumption of the preceding proposition, so this result is a special case of our proposition. The same referee remarked that a book of Belousov and Andronov [9], which unfortunately is not accessible to us, proves another existence result similar to the preceding proposition, but with $X=\mathbb{R}^{n}$ and each convex $f_{j}: \mathbb{R}^{n} \rightarrow \mathbb{R}$ having the property that, for each $d \in \mathbb{R}^{n}$, either

$$
\lim _{\alpha \rightarrow \infty} \frac{f_{j}(x+\alpha d)}{\alpha}=\infty, \quad \forall x \in \mathbb{R}^{n}
$$

or, for some $\mu_{j} \in \mathbb{R}$ (depending on $d$ ),

$$
f_{j}(x+\alpha d)=f_{j}(x)+\mu_{j} \alpha, \quad \forall \alpha \in \mathbb{R}, \quad \forall x \in \mathbb{R}^{n} .
$$

This result is again a special case of Proposition 11 since it can be verified that each such $f_{j}$ (which includes convex polynomials) is bidirectionally flat. ${ }^{1}$ The converse is not true. The Euclidean norm function is bidirectionally flat, but does not have the above property.

We now consider another type of direction, which when used in conjunction with horizon directions, yields some conditions that slightly improve on the conditions of Proposition 10. Let $f: \mathbb{R}^{n} \mapsto(-\infty, \infty]$ be a closed proper convex function, and let $F_{f}$ be the set of all directions $y$ such that for every $x \in \operatorname{dom}(f)$, the $\operatorname{limit}_{\alpha \rightarrow \infty} f(x+\alpha y)$ exists and is finite. Intuitively, $F_{f}$ is the set of directions along which $f$ is "flat". Note that

$$
L_{f} \subset F_{f} \subset R_{f} .
$$

We have the following variant of Proposition 10.

Proposition 12 (Convex Cost Function). Let $f: \mathbb{R}^{n} \mapsto(-\infty, \infty]$ be a closed proper convex function, and let $X$ be a closed set whose asymptotic directions are retractive local horizon directions. Assume that

$$
A_{X} \cap F_{f} \subset L_{f}
$$

Then $f$ attains a minimum over $X$ if and only if the optimal value $\inf _{x \in X} f(x)$ is finite.

Proof. Assume that the optimal value is finite. Then $X \cap \operatorname{dom}(f) \neq \emptyset$. Let $d \in A_{X} \cap R_{f}$. If $d \notin F_{f}$, then we must have $\lim _{\alpha \rightarrow \infty} f(x+\alpha d)=-\infty$, for some $x \in \operatorname{dom}(f) \cap X$. Since $d$ is a local horizon direction of $X$, we have $x+\alpha d \in X$ for all $x \in X$ and sufficiently large $\alpha$. It follows that $\inf _{x \in X} f(x)=-\infty$, a contradiction. Therefore, we must have $A_{X} \cap F_{f}=A_{X} \cap R_{f}$, so using the hypothesis, we obtain $A_{X} \cap R_{f} \subset L_{f}$. From Proposition 10, it follows that there exists at least one minimizing point.

\footnotetext{
${ }^{1}$ If $d$ satisfies Eq. (2), then Eq. (12) cannot hold, so Eq. (13) must hold for some $\mu_{j} \in \mathbb{R}$. Then $\lim _{\alpha \rightarrow \infty} f_{j}(x+\alpha d) / \alpha=\mu_{j}$ so Eq. (2) implies $\mu_{j}=0$ and Eq. (13) yields $d \in L_{f_{j}}$. Conversely, if $d \in L_{f_{j}}$, then Eq. (13) holds with $\mu_{j}=0$, and Eq. (2) follows.
} 
The following proposition extends a classical result, known as the Frank-Wolfe Theorem [16], which states that every (possibly nonconvex) quadratic programming problem has an optimal solution if and only if it has finite optimal value. Alternative proofs of this result are given in $[12,15]$, and various extensions are given in $[8,10,19$, $21,24]$. The following proposition becomes the Frank-Wolfe Theorem in the special case where $f$ is a quadratic function and the constraint set $X$ is a polyhedral set.

Proposition 13 (Extended Frank-Wolfe Theorem). Let $f: \mathbb{R}^{n} \mapsto(-\infty, \infty]$ be $a$ closed proper function, and let $X$ be a closed set such that $X \cap \operatorname{dom}(f) \neq \emptyset$. Assume that:

(1) All the asymptotic directions of $X$ are retractive local horizon directions.

(2) For every decreasing scalar sequence $\left\{\gamma_{k}\right\}$ such that the sets

$$
S_{k}=X \cap\left\{x \mid f(x) \leq \gamma_{k}\right\}, \quad k=0,1, \ldots,
$$

are nonempty, for every asymptotic direction $d$ of $\left\{S_{k}\right\}$, and for each $x \in X$, we either have $\lim _{\alpha \rightarrow \infty} f(x+\alpha d)=-\infty$, or else $f(x-d) \leq f(x)$.

Then $f$ attains a minimum over $X$ if and only if the optimal value $\inf _{x \in X} f(x)$ is finite.

Proof. Assume that $f^{*}=\inf _{x \in X} f(x)$, the optimal value, is finite. Let $\left\{\gamma_{k}\right\}$ be a scalar sequence with $\gamma_{k} \downarrow f^{*}$, and denote

$$
V_{k}=\left\{x \mid f(x) \leq \gamma_{k}\right\},
$$

so that $S_{k}=X \cap V_{k}$. It will suffice to show that each asymptotic direction $d$ of $\left\{S_{k}\right\}$ is retractive for $\left\{V_{k}\right\}$ (since $d$ is also retractive for $X$ by assumption, this shows that $d$ is retractive for $\left\{S_{k}\right\}$, and Proposition 1 applies).

Indeed, if $d$ is an asymptotic direction of $\left\{S_{k}\right\}$, then $d$ is a local horizon direction of $X$, so that for each $x \in X$ and all $\alpha$ sufficiently large, $x+\alpha d \in X$ and hence $f(x+\alpha d) \geq f^{*}$. Thus, we cannot have $\lim _{\alpha \rightarrow \infty} f(x+\alpha d)=-\infty$, and from our assumptions, it follows that $f(x-d) \leq f(x)$ for all $x \in X$.

Now consider the asymptotic sequence $\left\{x_{k}\right\}$ corresponding to the asymptotic direction $d$ of $\left\{S_{k}\right\}$. For all $k, f\left(x_{k}-d\right) \leq f\left(x_{k}\right)$. Since $x_{k} \in V_{k}$, this shows that $x_{k}-d \in V_{k}$ and hence $d$ is retractive for $\left\{V_{k}\right\}$. Since $d$ is also an asymptotic direction of $X$ and hence is retractive for $X$, it follows that $d$ is retractive for $\left\{S_{k}\right\}$.

The preceding proof can be used to show the result under a slightly weaker assumption: one may assume that only those asymptotic directions of $X$ that are also asymptotic directions of some level set of $f$ (rather than all asymptotic directions of $X$ ) are retractive local horizon directions of $X$.

The assumption (2) in Proposition 13 is satisfied if $f$ is a real-valued concave function, as is easily verified. It is also satisfied if $f$ is a quadratic function

$$
f(x)=x^{\prime} Q x+c^{\prime} x, \quad \forall x \in \mathbb{R}^{n},
$$

where $Q$ is a real symmetric $n \times n$ matrix, and $c$ is a vector in $\mathbb{R}^{n}$. Indeed, every asymptotic direction $d$ of $\left\{x \mid f(x) \leq \gamma_{k}\right\}$, where $\left\{\gamma_{k}\right\}$ is a decreasing scalar sequence, satisfies

$$
d^{\prime} Q d \leq 0 .
$$


Thus, for each $x \in \mathbb{R}^{n}$, either $(c+2 Q x)^{\prime} d<0$, in which case

$$
f(x+\alpha d)=f(x)+\alpha(c+2 Q x)^{\prime} d+\alpha^{2} d^{\prime} Q d \rightarrow-\infty \text { as } \alpha \rightarrow \infty,
$$

or else $(c+2 Q x)^{\prime} d \geq 0$, in which case

$$
f(x-d)=f(x)-(c+2 Q x)^{\prime} d+d^{\prime} Q d \leq f(x) .
$$

In the case where $X=X_{1} \cap X_{2} \cap \cdots \cap X_{m}$, with each $X_{i}$ being the vector sum of a compact set and a polyhedral cone $N_{i}$ (e.g. when $X$ is a polyhedral set), all asymptotic directions of $X$ are retractive local horizon directions (see Example 3). Thus, the assumption on $X$ of the preceding proposition is satisfied, and there exists an optimal solution when the optimal value is finite. This extended version of the Frank-Wolfe Theorem is credited to Kummer [19] by Belousov and Klatte [10] for the case of quadratic $f$, and to Belousov [8] by a referee for the case of concave $f$. A different extension was given by Perold [24], where $X$ is a polyhedral set and $f$ belongs to a class $\mathcal{G}$ which generalizes quadratic functions, concave functions, and coercive functions. The extended FrankWolfe Theorem given here is, of course, more general. For example, it applies to some situations where the constraint set is defined by concave inequalities (see Proposition 4, Example 7). In particular, a concave or a quadratic cost function attains a minimum over a set $X$ defined by linear or strictly concave quadratic inequalities:

$$
X=\left\{x \mid x^{\prime} Q_{j} x+c_{j}^{\prime} x+b_{j} \leq 0, j=1, \ldots, r\right\},
$$

where each matrix $Q_{j}$ is either equal to 0 or is a negative definite matrix (see Example 7).

Besides concave and quadratic functions, there are other functions that satisfy assumption (2) of Proposition 13, which do not seem to have been considered previously. One such function is of the form

$$
f(x)=p\left(x^{\prime} Q x\right)+c^{\prime} x+b,
$$

where $Q$ is a positive semidefinite matrix, $c$ is a vector, $b$ is scalar, and $p(\cdot)$ is a polynomial. Indeed, assumption (2) clearly holds if $p$ is a constant. If $p$ is not a constant, for any asymptotic direction $d$ of $\left\{S_{k}\right\}$, there are two cases: (1) $Q d \neq 0$, in which case the highest degree term in $p(\cdot)$ has a negative coefficient and hence $\lim _{\alpha \rightarrow \infty} f(x+\alpha d)=-\infty$; (2) $Q d=0$, in which case either $\lim _{\alpha \rightarrow \infty} f(x+\alpha d)=-\infty$ or $f(x+\alpha d)$ is a nondecreasing function of $\alpha$, depending on whether $c^{\prime} d<0$ or $c^{\prime} d \geq 0$. Thus, assumption (2) again holds.

Another example is a polynomial function $f$ that is quasiconvex over $X$, i.e. the intersection of $X$ with each level set of $f$ is convex. Indeed, for any asymptotic direction $d$ of $\left\{S_{k}\right\}$ and each $x \in X$, we have that $x \in S$ and $\left\{x_{k}\right\} \subset S$, where $S_{k}$ is defined as in assumption (2), $\left\{x_{k}\right\}$ is any asymptotic sequence corresponding to $d$, and

$$
S=X \cap\left\{y \mid f(y) \leq \max \left\{f(x), \gamma_{0}\right\}\right\} .
$$

Since $S$ is convex by our assumption, $d$ is a recession direction of $S$, so that $x+\alpha d \in S$ for all $\alpha>0$. Then $f(x+\alpha d) \leq \max \left\{f(x), \gamma_{0}\right\}$ for all $\alpha>0$. Since $f(x+\alpha d)$ is a 
polynomial function of $\alpha$, this implies that either $\lim _{\alpha \rightarrow \infty} f(x+\alpha d)=-\infty$ or else $f(x+\alpha d)$ is a constant function of $\alpha$. Thus, assumption (2) holds.

A referee remarked that an extension of the Frank-Wolfe Theorem was proved in [9] for the problem of minimizing a cubic function over a polyhedral set (also see the discussion in [10], page 38). The same referee noted another extension of the FrankWolfe Theorem, given in Theorem. 4.5.4 of [5] and also in Theorem. 2.2 of [6], whereby the Hessian of the quadratic function and the left-hand constraint matrix are rational and some of the variables are constrained to be integer-valued. A weaker version of this result that assumes all problem data are rational was proved earlier by Mandel [22]. Can related results be proved by using the ideas in this paper? We leave this as a topic for future research.

Acknowledgements. Thanks are due to Huizhen Yu for helpful interactions on the subject. Thanks are also due to two referees for their helpful comments and detailed suggestions on earlier versions of this paper. Dimitri P. Bertsekas was supported by NSF Grant ECS-0218328. Paul Tseng was supported by NSF Grant DMS-0511283.

\section{References}

1. Auslender, A., Teboulle, M.: Asymptotic Cones and Functions in Optimization and Variational Inequalities. Springer, Berlin Heidelberg New York (2003)

2. Auslender, A.: Non coercive optimization problems. Math. Oper. Res. 21, 769-782 (1996)

3. Auslender, A.: How to deal with the unbounded in optimization: theory and algorithms. Math. Program. 79, 3-18 (1997)

4. Auslender, A.: Existence of optimal solutions and duality results under weak conditions. Math. Program. 88, 45-59 (2000)

5. Bank, B., Guddat, J., Klatte, D., Kummer, B., Tammer, K.: Nonlinear Parametric Optimization. Birkhäuser Verlag, Basel-Boston (1983)

6. Bank, B., Hansel, R.: Stability of mixed-integer quadratic programming problems. Math. Program. Study 21, 1-17 (1984)

7. Bank, B., Mandel, R.: Parametric Integer Optimization. Akademie-Verlag, Berlin (1988)

8. Belousov, E.G.: Introduction to Convex Analysis and Integer Programming (in Russian). Moscow University Publishers, Moscow (1977)

9. Belousov, E.G., Andronov, V.G.: Solvability and Stability of Problems of Polynomial Programming (in Russian), Moscow University Publishers, Moscow (1993)

10. Belousov, E.G., Klatte, D.: A Frank-Wolfe type theorem for convex polynomial programs. Comput. Optim. Appl. 22, 37-48 (2002)

11. Bertsekas, D.P., with Nedić, A., Ozdaglar, A.E.: Convex Analysis and Optimization. Athena Scientific, Belmont (2003)

12. Blum, E., Oettli, W.: Direct proof of the existence theorem for quadratic programming. Oper. Res. 20, 165-167 (1972)

13. Dedieu, J.P.: Cone asymptotiques d'un ensemble non convexe. application a l' optimization. C.R. Acad. Sci. 287, 91-103 (1977)

14. Dedieu, J.P.: Cones asymptotiques d' ensembles non convexes. Bulletin Societe Mathematiques de France, Analyse Non Convexe, Memoire 60, 31-44 (1979)

15. Eaves, B.C.: On quadratic programming. Management Sci. 17, 698-711 (1971)

16. Frank, M., Wolfe, P.: An algorithm for quadratic programming. Naval Res. Logistics Quart. 3, 95-110 (1956)

17. Fenchel, W.: Convex Cones, Sets, and Functions. Mimeographed Notes, Princeton University, Princeton (1951)

18. Helly, E.: Uber Systeme Linearer Gleichungen mit Unendlich Vielen Unbekannten. Monatschr. Math. Phys. 31, 60-91 (1921).

19. Kummer, B.: Globale Stabilität quadratischer Optimierungsprobleme. Wissenschaftliche Zeitschrift der Humboldt-Universität zu Berlin, Math.-Nat. R. XXVI, 565-569 (1977) 
20. Kummer, B.: Stability and weak duality in convex programming without regularity. Wissenschaftliche Zeitschrift der Humboldt-Universität zu Berlin, Math.-Nat. R. XXX, 381-386 (1981)

21. Luo, Z.-Q., Zhang, S.Z.: On the extension of Frank-Wolfe theorem. Comput. Optim. Appl. 13, 87-110 (1999)

22. Mandel, R.: Über die Existenz von Lösungen ganzzahliger Optimierungsaufgaben. [On the Existence of Solutions of Integer Programming Problems] Math. Operationsforsch. Statist., Ser. Optim. 12, 33-39 (1981)

23. Pataki, G.: On the closedness of the linear image of a closed convex cone. Research Report TR-02-3, Department of Operations Research, University of North Carolina, Chapel Hill (2003)

24. Perold, A.F.: A generalization of the Frank-Wolfe theorem. Math. Program. 18, $215-227$ (1980)

25. Rockafellar, R.T.: Convex Analysis. Princeton University Press, Princeton (1970)

26. Rockafellar, R.T.: Ordinary convex programs without a duality gap. J. Optim. Theory Appl. 7, 143-148 (1971)

27. Rockafellar, R.T., Wets, R.J.-B.: Variational Analysis. Springer, Berlin Heidelberg New York (1998)

28. Terlaky, T.: On $l_{p}$ programming. Euro. J. Oper. Res. 22, 70-100 (1985)

29. Tseng, P., Ozdaglar, A.E.: Existence of global minima for constrained optimization. J. Optim. Theory Appl. 128, (2004) (to appear) 